\title{
Balkanologie
}

Balkanologie Revue d'études pluridisciplinaires

Vol. I, n 1 | 1997

Volume I Numéro 1

\section{Les laboratoires yougoslave et caucasien}

État, développement et ré-ajustement global dans l'après guerre froide

\section{Ivan Iveković}

Traducteur : Sylvie Pomiès

\section{OpenEdition}

Journals

Édition électronique

URL : http://journals.openedition.org/balkanologie/198

DOI : 10.4000/balkanologie. 198

ISSN : 1965-0582

\section{Éditeur}

Association française d'études sur les Balkans (Afebalk)

Édition imprimée

Date de publication : 1 juillet 1997

ISSN : 1279-7952

\section{Référence électronique}

Ivan Iveković, « Les laboratoires yougoslave et caucasien », Balkanologie [En ligne], Vol. I, n 1 | 1997, mis en ligne le 02 juin 2008, consulté le 17 décembre 2020. URL : http://journals.openedition.org/ balkanologie/198; DOI : https://doi.org/10.4000/balkanologie.198

Ce document a été généré automatiquement le 17 décembre 2020.

(ㄷ) Tous droits réservés 


\title{
Les laboratoires yougoslave et caucasien
}

\author{
État, développement et ré-ajustement global dans l'après guerre froide
}

\section{Ivan Iveković}

Traduction : Sylvie Pomiès

\section{NOTE DE L'ÉDITEUR}

Ce texte a été traduit de l'anglais par Sylvie Pomiès.

\section{Introduction}

1 Dans les temps modernes, un lien étroit s'est établi entre l'État, la contrainte étatique et le développement économique, en dépit de la minimisation systématique du problème par les défenseurs acharnés de la libre entreprise et du "laisser faire". Contrariés par la nature "totalitaire" de l'État communiste, et par son ambition de promouvoir le développement "par en haut" et de présenter l'action économique de l'État comme une alternative d'ensemble au mode de production capitaliste, les partisans néo-libéraux du libre échange appelèrent au "retrait » de l'État dans leurs propres pays et combattirent, pendant toute la période de la guerre froide, non seulement l'alliance d'États dirigée par l'Union soviétique, mais également l'institution de l'État en tant que tel, perçu comme le principal ennemi de la liberté de l'individu, de la prospérité et de la démocratie. C'est pendant cette période que la théorie de l'État en tant que sous-discipline des sciences sociales a été quelque peu marginalisée ${ }^{1}$. Le rôle intérieur de l'État pendant les années de formation des sociétés industrielles modernes et dans la protection de l'industrie "nationale" naissante face à la compétition étrangère ont été complètement oubliés. De même, on a délibéremment ignoré le fait que les modèles économiques libéraux les plus florissants ont été eux-mêmes indirectement ou même directement subventionnés par leurs propres États (achat 
d'armes, subventions pour l'agriculture) ou bien protégés par toutes sortes d'obstacles à l'encontre des pays en compétition (traitements préférentiels, quotas, barrière à l'immigration économique). Le fait que les démocraties libérales aient pris part aux principales guerres internationales, des guerres de conquête et d'expansion de leur marché et de leurs zones d'influence, a été négligé. Cependant, les études sur le développement traitant des problèmes du Tiers Monde ont replacé l'État sur le devant de la scène. On a compris que son rôle ne pouvait être évité et que les lois de l'économie de marché, seules, ne suffisaient pas à promouvoir le "développement" (quelque soit le sens donné à ce terme). On a aussi reconnu que l'État, avec son monopole de coercition, proche de la violence d'État des régimes communistes "totalitaires", a joué un rôle fondamental dans l'étape de décollage économique des pays en voie de développement d'Asie. Après l'échec du projet communiste dans l'ancienne Union soviétique et en Europe centre-orientale, l'État est une fois de plus appelé à remplir le rôle clef dans le tranfert des mesures économiques prises par lui, dans le processus de privatisation et dans celui d'intégration des économies "nationales" radicalement restructurées dans le système global.

2 Il n'est pas dans mes intentions de renforcer la théorie de l'État, mais d'analyser de manière plus pragmatique les relations entre l'ethnicité, le nationalisme, l'État-nation, l'économie politique globale et la hiérarchie changeante de la puissance internationale (changement de pouvoir) comme elles étaient et sont perçues dans les "laboratoires régionaux" de Yougoslavie et du Caucase. Nous accorderons plus d'importance aux pays suivants :

- la République fédérale de Yougoslavie croupion (Serbie et Monténégro) : population totale de 10406000 ; composition ethnique : 62,3\% de Serbes, 16,6 \% d'Albanais, 3,3\% de Hongrois, 3,3 \% de Yougoslaves, 3,1\% de Musulmans (1991) ;

- la Croatie : population de 4782000 ; composition ethnique : 78,11\% de Croates, $12,1 \%$ de Serbes, autres : $9,8 \%$ (1991);

- Arménie : population de 3287000 ; composition ethnique : 91 \% d'Arméniens, 3 \% d'Azéris, $3 \%$ de Kurdes, 3 \% de Russes (1991) ;

- Azerbaïdjan : population de 7037000 ; composition ethnique : 83 \% d'Azéris, $6 \%$ d'Arméniens, 6 \% de Russes (1991).

Le concept de "laboratoires régionaux" est géostratégique. L'ancienne Yougoslavie avec sa politique de non-alignement constituait une zone tampon entre les deux blocs ennemis et le Caucase formait la périphérie de l'ancienne Union soviétique et sa zone frontalière avec le Moyen-Orient. Avec la décomposition des deux fédérations multiethniques et leur remplacement par un grand nombre de nouvelles entités étatiques officiellement indépendantes, les deux "laboratoires" ont été transformés en enclaves de guerre dans lesquelles les pouvoirs locaux, régionaux et extérieurs testent leurs propres capacités et les intérêts et aptitudes de leurs rivaux réels ou potentiels, et tentent de définir leur politique stratégique à long terme. Nous parlons ici de deux zones d'ensembles géographiques délimités et distincts devant aujourd'hui affronter des problèmes similaires : la sortie du communisme, les troubles internes, les conflits ethniques, la violence politique et les guerres territoriales. Ces dernières années, les deux "laboratoires" ont été, de manière tout à fait inattendue, "balkanisés" ou "libanisés". Les rivaux croates et serbes dans les Balkans et les ennemis jumeaux Arméniens et Azéris dans le Caucase ne sont que des morceaux de patchworks 
ethniques plus vastes. Pour couvrir l'ensemble de la situation existant dans les deux laboratoires, il faut également aborder la question des tensions et des conflits locaux :

- mise à part la guerre fratricide en Bosnie-Herzégovine (43,7 \% de Slaves musulmans, $31,4 \%$ de Serbes, 17,3 \% de Croates, 5,5 \% de Yougoslaves en 1991), toutes les anciennes républiques, à l'exception de la Slovénie, ont aussi des problèmes avec les minorités nationales; environ la moitié des Albanais vivent en dehors de l'Albanie et souffrent de discrimination (la crise yougoslave a commencé au Kosovo où les Albanais représentent plus de $90 \%$ de la population locale) ; la Grèce de Papandréou ne reconnaît toujours pas l'existence de minorités sur son territoire et ne s'entend pas avec tous ses voisins ; la Bulgarie souffre de problèmes non-résolus avec sa minorité turque et musulmane et la Roumanie avec les Hongrois de Transylvanie ; la Moldavie est confrontée à la séparation de la République du Dniestre (Russes et Ukrainiens) et à sa minorité turque gagaouze ; la Turquie combat la rébellion kurde, particulièrement bien organisée en Anatolie orientale (et récemment dans le nord de l'Irak) et a une minorité religieuse alaouite qui commence à bouger ;

- la situation n'est pas meilleure dans le "laboratoire" du Caucase où la guerre pour le contrôle du Haut-Karabagh (123000 Arméniens et 37000 Azéris en 1979) a éclaté avant l'implosion de l'URSS ; à cause d'une fracture interne, l'Azerbaïdjan a perdu près du tiers de son territoire au profit des "volontaires" arméniens ; l'Ossétie du Sud (66 \% d'Ossètes, 28 \% de Géorgiens) et l'Abkhazie ( $18 \%$ d'Abkhazes et $48 \%$ de Géorgiens) se sont pratiquement séparés de la Géorgie indépendante, qui elle-même sombre dans une lutte sanglante pour le pouvoir entre plusieurs clans ; l'ensemble du Caucase du nord, officiellement partie intégrante de la fédération russe, est déchiré par des tensions et des conflits ethniques, et la récente expédition militaire russe contre la séparation de la Tchétchénie va très certainement ajouter de l'huile sur le feu.

\section{Modèles de comparaison}

4 Comme nous abordons les développements post-communistes dans ces deux régions, nous devons tout d'abord comprendre et identifier leurs origines spécifiques. En d'autres termes, nous devons remonter aux périodes pré-communiste et communiste.

5 La Russie tsariste et le Royaume de Yougoslavie étaient des autocraties traditionnelles et des États unitaires comprenant des populations ethniquement mélangées. Les sujets non-Russes et non-Serbes considéraient l'autorité centrale de l'État comme un corps étranger - à savoir comme une occupation étrangère. Lénine surnomma la Russie "prison des peuples" et le Parti communiste yougoslave évoquait la monarchie yougoslave dans des termes similaires. L'économie de ces deux pays multiethniques était essentiellement agraire, cependant, il faut noter que la zone pétrolifère de Bakou au début du XX⿳亠丷⿵冂丶 ${ }^{m e}$ siècle et les industries de Zagreb à la veille de la Seconde Guerre mondiale représentaient peut-être, malgré leur position isolée, les pôles de développement moderne les plus importants dans ces deux pays.

D'après la définition classique proposée par Friedrich et Brzezinski, les systèmes communistes qui devaient plus tard s'imposer étaient totalitaires. Avec les régimes nazis et fascistes, ils partageaient les caractéristiques suivantes :

- une idéologie totalitaire officielle, couvrant tous les aspects de la vie quotidienne, caractérisée par la soumission catégorique ; toute personne vivant dans cette société devait adhérer aux normes officielles ; 
- un parti unique et puissant, dirigé par un seul homme, le dictateur, complémentaire et inséparable de l'appareil du gouvernement et exerçant un monopole sur toutes les activités politiques ;

- un système de contrôle de police fondé sur la terreur et l'intimidation, utilisant des techniques de surveillance et de torture modernes;

- un monopole technologique concentré au sein du parti ou dans les mains du dictateur contrôlant tous les mass-médias ;

- une direction et un contrôle central de tous les secteurs de l'économie à travers la coordination bureaucratique de toutes les associations lucratives anciennement indépendantes et, plus précisément, les groupes et les activités collectives ;

- parmi ces caractéristiques figure également le monopole de l'État sur les moyens de défense et de combat armé ${ }^{2}$.

Le totalitarisme était un concept essentiellement associé à la pratique de Staline. Malheureusement pour Friedrich et Brzezinski, ceci ne correspondait plus à l'évolution du modèle après la mort de Staline. En fait, le modèle stalinien avait un certain nombre de contre-modèles. Le processus de déstalinisation fit place à ce qu'Adam Michnik en Pologne appela le «totalitarisme aux dents limées ${ }^{3}$ ou au «totalitarisme bienveillant " comme le décrivit Vesna Pusic en Yougoslavie ${ }^{4}$. En effet, il y avait un grand fossé entre le régime génocidaire de Pol Pot au Cambodge d'un côté, et le "socialisme au goulash» hongrois très permissif de l'autre. Le juste milieu correspondait au réel-socialisme brejnévien ${ }^{5}$. En d'autres termes, les variations "nationales" étaient révélatrices. De plus, ce "monopole d'État des moyens de lutte ", mentionné ci-dessus, n'est pas une caractéristique spécifique au totalitarisme : c'est la prérogative de tous les États, y compris les plus démocratiques.

Cependant, il y avait un modèle général de direction communiste reproduit dans tous les pays du "socialisme réel" :

- une « idéologie objective de la classe ouvrière » totalitaire interprétée par les élites communistes dirigeantes (ayant aboutit à diverses voies vers le socialisme) ;

- un État-parti monopolisant les sphères de la politique et de la vie publique (la clause constitutionnelle concernant le "rôle dirigeant" du Parti communiste), mais permettant toutefois l'émergence de sociétés civiles dans sa phase de déclin;

- une économie dirigée par l'État - finalement toutes les réformes marchandes partielles de l'économie de marché ont échoué en Europe de l'est, et il reste à prouver qu'elles puissent fonctionner en République populaire de Chine ;

- une bureaucratie étatique gigantesque, avec sa nomenklatura, exploitant la majorité de la population;

- une ingérence sélective de l'État-parti dans la vie privée des citoyens ;

- un monopole de l'État-parti sur les mass-médias, qui, peut-être par nécessité, s'est progressivement affaibli avec la révolution des moyens d'information et la crise interne générale du système ;

- pour les États multiethniques seulement : une administration territoriale fédérale "à la Lénine" et une question nationale non-résolue.

Cette conceptualisation s'apparente beaucoup à la définition proposée par Friedrich et Brzezinski ; toutefois, elle est plus générale et moins stricte. Elle tient compte à la fois de l'évolution du modèle à l'intérieur de chaque pays et des variations locales, nationales, voire régionales. 
10 Maintenant, en dépit de leurs différences, on peut citer les traits communs existant entre les États et les "nouvelles" sociétés caucasiennes et balkaniques :

- les systèmes politiques et économiques sont encore en état de fluctuation et de transition ;

- il s'agit plutôt de la domination d'une "mentalité" nationaliste que celle d'une idéologie

"totale";

- les minorités nationales sont de jure et / ou de facto exclues du système. Elles sont particulièrement victimes du régime de terreur, de la discrimination et de la purification ethnique organisée par l'État nationaliste ;

- un pluralisme politique à dimension unique : la "scène politique nationale" étant rythmée par de grands mouvements électoraux ou des coalitions parlementaires éphémères, principalement dirigées par des leaders charismatiques du "type caudillo"; machines de vote, marchandage entre élites et politique de la clique ;

- la classe dirigeante exerce son pouvoir avec des limites mal définies mais souvent prévisibles ; de fortes tendances autocratiques, mais pas de contrôle total de la vie publique ; le dirigeant arbitre entre les cliques par cooptation et corruption ; rapide "circulation d'élites" ;

- manipulations de l'État sur - et par - les mass-médias, surtout la télévision, la radio et la presse à grands tirages, particulièrement surveillés par les cercles proches du régime ;

- ces États sont “faibles" et peu légitimes, ils s'appuient spécialement sur les moyens illégitimes de violence afin de terroriser le segment de population visé. Employant des moyens illégaux, des milices privées et, en dernière instance, des troupes de mercenaires peu fiables, un climat d'insécurité interne se crée, allant même parfois jusqu'à mettre le régime lui-même en danger ${ }^{6}$.

De tels systèmes peuvent être qualifiés d'“ethnocraties autoritaires", de "démocraties ethniques dictatoriales", de "régimes nationalistes post-communistes", ou bien encore de "dictature à légitimation électorale". La Géorgie, la Moldavie, la Slovaquie et d'autres pays pourraient encore entrer dans cette catégorie. Ils forment déjà un groupe bien distinct parmi les sociétés post-communistes et tranchent singulièrement avec les "démocraties non-consolidées" qui prennent corps en Pologne, dans la République tchèque, en Hongrie et en Slovénie. Ils doivent aussi être distingués des régimes "crypto-communistes" ou des "autocraties post-communistes modernes" avec lesquels ils partagent des caractéristiques communes ${ }^{7}$. Quelles que soient les caractéristiques des différents régimes post-communistes, ils ont tous à leur ordre du jour la transition vers l'économie de marché et la démocratie. Il s'agit de toute évidence d'un processus qui s'annonce douloureux, tortueux et fortement aléatoire.

\section{Économie de marché et changement de régime}

Pour mieux comprendre les obstacles objectifs et subjectifs à une transition rapide et en douceur vers l'économie de marché, nous devons tout d'abord revenir sur quelques aspects essentiels de l'économie étatique :

- de fait, l'État-parti était propriétaire de tous les moyens de production les plus importants et l'unique distributeur et allocataire des rares ressources ; c'était également le principal employeur ;

- le contrôle, la coordination et la planification centrale administrative avaient remplacé de fait la libre-circulation des capitaux, des biens et de la main-d'oeuvre ; 
- comme les allocations étaient distribuées de haut en bas selon la division territoriale hiérarchique, l'économie était territorialisée (immobilisée), le centre jouant le rôle d'arbitre entre les différentes unités territoriales en compétition ;

- l'économie dirigée avait créé une bureaucratie parasite de l'État-parti et une nomenklatura qui lui était propre ;

- les défauts du système et le climat plus ou moins permissif avait favorisé la croissance d'une seconde économie (fonctionnant selon la logique du marché) ayant, peu à peu, affaibli l'économie officielle de l'intérieur ;

- les demi-réformes accordant aux entreprises "socialistes" une certaine marge d'autonomie (la plus développée étant le Nouveau Mécanisme Économique Hongrois) visaient à la décentralisation territoriale (cf. ancienne Yougoslavie). Finalement, les mesures furent gelées par la lutte pour le pouvoir entre les communistes réformateurs et les conservateurs ;

- la bousculade pour la technologie et les prêts occidentaux endetta les pays communistes et devint un fardeau pour leurs sociétés.

Dans les économies anciennement agraires, le modèle soviétique de l'accumulation du capital a été reproduit pratiquement partout. La terre a été collectivisée et, grâce à un mécanisme de prix artificiels, le surplus issu du secteur agricole était orienté vers l'industrialisation rapide ${ }^{8}$. L'accent mis sur l'énergie, les infrastructures et l'industrie lourde - la soi-disant "base matérielle du développement socialiste" - devait à son tour, selon la logique interne, donner naissance au travailleur socialiste moderne et à la classe industrielle moderne au nom de laquelle le parti avait pris le pouvoir.

La nourriture bon marché et les prix surélevés des produits manufacturés étaient censés ajouter à la liste des motivations, bien que les salaires des ouvriers soient restés bas. Le concept général de développement était autarcique et en principe, sinon en pratique, l'idée était de créer des unités territoriales économiquement auto-suffisantes et au développement harmonieux ${ }^{9}$. Employant une main d'œuvre extensive et orientant les investissements, une telle politique "modernisa" ces sociétés anciennement arriérées économiquement, et put se vanter de résultats quantitatifs. En 1961, le PCUS (Parti Communiste de l'Union Soviétique) adopta le plan mégalomane de Khroutchev, basé sur un calendrier bien précis devant déboucher sur la transition au communisme et le "dépassement" économique des États-Unis, dans un délai de vingt ans. Trois ans auparavant, la Ligue des Communistes de Yougoslavie (LCY) avait promulgué son propre programme d'“autogestion sociale" tout aussi optimiste, qui devait servir de référence pour beaucoup de réformateurs communistes est-européens.

Nous savons désormais que la bureaucratie soviétique et le "socialisme à visage humain" yougoslave ont tous les deux échoué. Selon Daniel Chirots, leur échec est plus dû à leur propre "succès" qu'à leur défaut. Il soutient que les économies dirigées ont réussi à construire d'impressionant complexes chimiques et des aciéries, nécessitant beaucoup de main d'œuvre et, par ailleurs, très polluants, à l'image de ceux de Pittsburgh. Bakou en Azerbaïdjan, Erevan en Arménie, Sisak en Croatie, Zenica en Bosnie et Smederevo en Serbie correspondraient parfaitement à cette description. Malheureusement pour les planificateurs communistes, l'Occident hyper-industrialisé connaissait déjà une avance technologique d'au moins deux générations. La chronologie de Chirots retrace les différentes étapes technologiques du monde occidental. Tout commence par (1) les industries du coton du type Manchester des années 1780 aux années 1830 . Puis vint (2) l'ère du chemin de fer et du fer, entre 1840 et 1870, ensuite (3) entre les années 1870 et la Première Guerre mondiale, ce fut le tour de 
l'acier et du fer. Lors de la révolution d'octobre 1917, les bolcheviques furent visiblement inspirés par ces technologies très avancées. De plus, (4) aucune économie dirigée en Europe de l'est ne fut capable de maitriser la technologie de l'automobile et la pétrochimie qui s'étaient développées en Occident entre les années 1910 et 1970. La seule exception à la règle fut le complexe militaro-industriel de l'Union soviétique qui demeura isolé au sein de l'économie soviétique. Enfin, (5) alors que le Japon et l'Ouest confirmaient leur avance dans le domaine de l'électronique, de l'informatique et de la biotechonologie - ayant débuté dans les années 1970 -, les économies dirigées tentaient toujours de résoudre les problèmes sociaux engendrés par le retard économique le plus impressionnant au monde ${ }^{10}$. Ces "éléphants blancs socialistes" constituent en effet les plus lourds fardeaux légués aux régimes post-communistes.

16 Dans les années 1950, l'économie soviétique se développa et nécessita des facteurs de production plus variés et diversifiés. On commença également à réaliser que les réserves nationales de matières premières et de combustibles n'étaient pas éternelles et qu'une consommation effrenée pourrait conduire à un épuisement des gisements si des mesures concrètes n'étaient pas prises pour une exploitation plus rationnelle des ressources.

À cette époque, la compétition avec l'Ouest commençait à mettre la priorité sur l'innovation technologique et le renouvellement perpétuel de produits et de modes de production, de telle sorte qu'une plus grande production de mêmes produits ne pouvait garantir la prééminence de l'Union soviétique à l'avenir. Toutes ces demandes représentèrent un nouveau fardeau pour les planificateurs qui devaient faire face au problème insoluble des plans de coordination et d'enregistrement des données. Le plan lui-même cessa d'être le moteur du changement mais se révéla, de plus en plus, un frein au changement. Le système était maintenant ouvert à une remise en question fondamentale de ses principes de base. ${ }^{11}$

17 Dans le même temps, des économistes est-européens faisaient pression pour des réformes du système, dans le but de le rendre plus efficace économiquement, et plus dynamique d'un point de vue technologique. Tous les programmes de réformes économiques entrepris par les communistes tentèrent d'introduire des éléments marchands, mais les grandes lignes demeuraient "socialistes". L'idée principale était que l'État n'établirait que des "plans indicatifs", contrôlant l'ensemble de la structure de distribution des ressources d'investissement en conservant une stricte politique de prix et de revenus et le monopole d'État sur le commerce extérieur. Les entreprises, qui acquéraient ainsi une nouvelle autonomie, pouvaient réellement diriger leurs propres affaires. Le processus de prise de décision concernant l'investissement serait plus ou moins décentralisé, suivant les contours de la division territoriale administrative du pays. Les revenus et les salaires dépendraient principalement des profits des entreprises. Les expériences les plus poussées concernant les réformes de marché furent entreprises par la Yougoslavie et la Hongrie ; mais, le processus fut bloqué par la lutte pour le pouvoir entre les communistes conservateurs et les réformateurs. En voulant conserver son rôle décisionnel dans l'économie, craignant, par la suite, de perdre son monopole politique, la nomenklatura communiste signa son propre arrêt de mort.

18 À la base, la fracture entre les États fédéraux multiethniques est due à l'absence de la force intégrative d'une économie de marché interne.

En rejetant tout ce qui se rapportait au marché, le socialisme d'État délégait la totalité du fardeau de la gestion économique à l'État-parti. Ainsi, tout conflit révélé lié aux mauvais résultats économiques défie directement l'État et, par conséquent, 
l'ordre politique. Comme de nombreux régimes autoritaires et théocratiques du passé, sa capacité d'adaptation à un environnement qui récompense la flexibilité et le dynamisme est limitée. Les autorités centrales doivent faire des compromis avec les exigences locales, au point d'en devenir leurs prisonnières, un peu comme des acteurs qui cherchent à poursuivre leurs propres intérêts, même si cette démarche va en contradiction avec les stratégies adoptées par l'État. (...) La "corruption" a massivement remplacé la violence pour obtenir la soumission de la population. À cause d'une suppression sélective accrue des intérêts divers et de la sophistication des instruments de management, l'autonomie de la société augmenta tandis que l'ambition du régime à un contrôle total avait diminué. ${ }^{12}$

On pourrait aussi bien dire que la territorialisation de l'économie créa une sorte de "féodalisme communiste" où la relation entre le centre et la périphérie a pris la forme d'une hiérarchie dirigiste basée sur un système de patronage très personnalisé. L'Étatparti yougoslave sous Tito et l'État soviétique sous Brejnev jouaient le rôle d'allocateurs autoritaires de ressources. Leurs successeurs ne jouirent pas de la même autorité, et lorsque la dépression économique fit son apparition et que la réserve de ressources se tarrit, les nouveaux patrons furent incapables de satisfaire les attentes de leurs clients, mettant en question le système entier ${ }^{13}$. L'“économie négociée" nouvellement décentralisée entre les Républiques yougoslaves dégénéra en éternels marchandages. La lutte pour le pouvoir entre les élites républicaines qui s'en suivit ébranla la structure du patronage vertical et ouvrit la voie au "nationalisme bureaucratique" communiste ${ }^{14}$. De même, les tentatives de Gorbatchev de démanteler le réseau de patronage de Brejnev et à reconsidérer le système de distribution des ressources aux "Républiques parasitaires" de la périphérie déclencha un tollé général contre le "despotisme du centre" et précipita les demandes pour la souveraineté sur les ressources nationales ${ }^{15}$.

Le processus de "décomposition" des deux fédérations peut également être interprété comme une bousculade effrénée pour rétablir des sources alternatives de ressources. Le contrôle des ressources nationales, de l'économie et des finances, ainsi que l'accès direct aux investissements étrangers, étaient vitaux pour la survie des élites républicaines. Les barons des Républiques tentèrent de s'imposer comme des grands patrons souverains. Malheureusement pour eux, leur base politique était plutôt instable et leur légitimité contestée par les contre-élites nationalistes. Leur position était en même temps menacée par des forces extérieures, par l'État central qui détenait toujours le monopole de la violence, ou encore par des barons rivaux. Ils tentèrent de renforcer leur position en co-optant des contre-élites nationalistes et ouvrirent, ainsi, la voie au pluripartisme. Dans la foulée, les partis communistes respectifs perdirent leur identité initiale et furent radicalement transformés ou, tout simplement, évincés du pouvoir lors des élections libres.

21 Il faudrait peut-être aussi tenir compte de deux autres aspects importants :

- le rôle des différentes diasporas ethniques dans l'ancienne Yougoslavie et en Arménie ; elles investirent dans les partis nationalistes, leur procurant des programmes politiques irrédentistes et même du personnel ;

- au contraire des autres zones, l'économie souterraine avait déjà infiltré tous les domaines de la vie sociale, aussi bien en Yougoslavie que dans le Caucase, impliquant de larges couches de la population, ce qui ouvrit la voie, non seulement aux méthodes mafieuses (officielles et officieuses), mais aussi à un type spécifique de société civile et de dynamiques sociales.

22 Ajouté au type unidimensionnel de la "modernisation" communiste et de la stratification sociale, ces deux facteurs ont largement contribué à la configuration 
naissante des forces politiques et sociales. L'intelligentsia joua un rôle essentiel dans la création du nouveau programme nationaliste.

Les guerres ont bien sûr leurs propres dynamiques, logiques et économies politiques. $\mathrm{Si}$ les paramètres normaux du comportement économique et politique sont alternés en période de transition "normale", ils le sont bien plus lors de conflits armés partageant beaucoup de caractéristiques avec les guerres civiles classiques. Les États impliqués dans les conflits mobilisent toutes leurs ressources économiques et humaines pour l'effort de guerre, sous le prétexte que la fin justifie les moyens. Les revenus nationaux, l'argent des contribuables ou les contributions de donateurs étrangers et des diasporas sont détournés en fonds spéciaux pour l'achat d'armements, achats qui sont effectués, pour la plupart d'entre eux, dans des pays étrangers. Au nom de la suprématie de "l'intérêt national et de l'intérêt de l'État", les élites dirigeantes s'approprient ou se voient attribuer des pouvoirs discrétionnaires. Le contrôle parlementaire est soit fictif, soit suspendu.

$\mathrm{Du}$ fait de l'embargo officiel des Nations unies sur les importations d'armes des différents États issus de la Yougoslavie, ceux-ci ont établi des réseaux alternatifs "nonofficiels" pour l'approvisionnement en armes souvent liés à des pratiques criminelles et / ou à des groupes mafieux. Je suppose qu'il en va de même dans le Caucase. Comme d'habitude dans ce genre de situation, les "profiteurs de guerre", proches du pouvoir ou occupant des positions officielles, s'approprient la part du lion. Ce butin est déposé sur des comptes bancaires à l'étranger ou sert à acheter les actions des entreprises publiques privatisées à la va-vite. Le quartier général serbe pour le blanchiment de l'argent a été établi à Chypre, tandis que les courtiers croates opèrent depuis Frankfort, en Allemagne. La gestion de ces fonds est assurée par d'anciens communistes experts en commerce ou des hommes d'affaires dynamiques de la diaspora. Avec la perpétuelle "circulation des élites", les Ministres aux mandats brefs et leurs accolytes amassent des fortunes personnelles colossales en un rien de temps. Parfois, ceux qui étaient trop exposés fuyaient le pays, tandis que d'autres, dont les noms sont de notoriété publique, sont toujours en train, à l'heure actuelle, de dériver des capitaux pour l'effort de guerre et d'utiliser le processus de privatisation pour leur propre bénéfice. En fait, la poursuite de la guerre est dans leur intérêt. Malheureusement pour leur nation et leur pays, c'est ainsi que fonctionne le processus de réforme du marché. Une fois la guerre terminée et cette "accumulation primitive de capital" réalisée, ils pourront prendre leur retraite comme beaucoup de seigneurs de guerre libanais l'ont fait. Enfin, leurs enfants et petits-enfants deviendront des citoyens respectables, formant le noyau dur de la nouvelle classe d'entrepreneurs, préoccupés par la paix, la sécurité, le respect de la loi... et la démocratie.

Pour l'instant, et peut-être est-ce plus conforme aux pays post-communistes "conventionnels" ou "conformes", le modèle démocratique libéral, appuyé par les institutions monétaires internationales et dont le coût social peut paraître trop élevé, n'est pas forcément le mieux adapté au système politico-économique naissant. Les "thérapies de choc" pourraient fonctionner dans quelques pays proches de l'Union européenne, si l'Occident veut bien les aider financièrement. Autrement, pour une population déjà épuisée par une crise sociale et une économie maintenue tant bien que mal, de telles réformes seront plus douloureuses. De nombreux citoyens n'apprécient guère le déclin de l'État providence. Après l'apathie générale, une nostalgie procommuniste refait souvent surface. Si mon interprétation des dernières élections 
parlementaires en Lithuanie, en Pologne, en Hongrie et en Bulgarie est correcte, alors une approche plus gradualiste est déjà programmée. La plupart des élites postcommunistes "normales" sont de toute évidence attirées par l'État social ouesteuropéen, mais elles sont peut-être obligées, du moins durant la transition, d'opter pour le modèle des pays d'Asie retardataires qui combinent à la fois un pouvoir étatique fort, voire autoritaire, et un marché et une économie tournés vers l'exportation ${ }^{16}$.

Quelque soit leur choix, aujourd'hui seuls des groupes politiques marginaux déclarent ouvertement préférer un retour à une véritable économie dirigée socialiste. Il s'agit d'un modèle de développement économique qui a, ainsi que le parti-État et son idéologie "socialiste", perdu sa légitimité. Il ne s'agit plus d'un débat socialisme contre capitalisme, mais capitalisme pur et dur (brutal et primitif) contre capitalisme à visage humain. Ceci ne veut pas dire que les réflexes étatiques ont tout à coup disparu. En fait, ils sont appliqués sous l'étiquette de nationalisme économique, ayant lutté pour "l'indépendance et l'émancipation nationales" en coupant les liens économiques avec les ennemis et les voisins. Aujourd'hui, tous les frères-ennemis yougoslaves affirment leur désir d'intégration de leurs nouveaux États-nations à l'Europe, mais les dirigeants croates et serbes ne veulent rien entreprendre ensemble. À Zagreb, ma ville natale, le simple fait d'évoquer le désir d'une plus grande coopération économique balkanique relève, pour les officiels et leur propagande, de la trahison nationale et est interprété comme une tentative de raviver l'entité yougoslave haïe. Quant à savoir comment réconcilier le développement national autarcique avec les processus d'intégration globale, ils n'ont, bien sûr, aucune réponse. Les tendances étatiques sont aussi reproduites dans les configurations sociales héritées de l'ancien régime: la bureaucratie d'État, du moins son segment le moins adaptable, tente de sauvegarder ses privilèges dépassés; et les ouvriers, ayant voté en masse pour leurs partis et coalitions nationalistes, appellent désormais à la protection de leur État national contre la privatisation sauvage. Les protestations contre la "liquidation" du pays aux étrangers et les groupes mafieux sont très fortes, non seulement parmi ceux dont la position économique et sociale a été détériorée, mais également au sein des cercles gouvernementaux.

\section{Vers une ré-évaluation du rôle de l'État}

Qu'on le veuille ou non, l'État-nation moderne (dans le sens anglo-saxon du terme) est non seulement la forme d'organisation politique et économique la plus répandue dans la société contemporaine, mais aussi la plus inévitable. Selon Anthony Giddens, un Étatnation est un "réservoir délimité à pouvoir " $^{17}$ avec des citoyens et un territoire définis. Les États-nations sont des entités dans le système mondial qui poursuivent leurs buts en coopération ou en conflit entre eux. La plupart des théories reconnaissent que leur formation est directement liée à l'émergence du capitalisme en Europe occidentale et en Amérique du nord et à son extension progressive au reste du monde. Comme Giddens le souligne, ce sont «des unités administratives délimitées où les politiques adoptées par les gouvernements engagent des populations entières $»^{18}$. Ces unités jouent un rôle tout particulier dans la division internationale du travail et font partie du système inter-étatique hiérarchisé moderne. Cependant, le rôle de l'État a été systématiquement dénigré par les théories classiques d'origine marxiste et weberienne. 
Dans la tradition marxiste, l'État est un instrument d'exploitation des classes, un simple dérivé d'une certaine division de classe de la société. Il appartient à la "superstructure" et « disparaittra progressivement » dans un futur distant et sans classe. Dans la théorie non marxiste des groupes d'intérêt, l'État serait une « sorte d'arbitre neutre tranchant dans la lutte entre les groupes d'intérêt, mais n'est pas en lui-même l'acteur principal $\aleph^{19}$. En fait, Weber accordait plus d'importance à l'État, à la fois dans le processus d'accumulation du capital et dans la formation de la structure sociale, qu'il n'en paraît dans l'interprétation de Talcott Parsons de L'éthique protestante et l'esprit du capitalisme, qui est, en grande partie, responsable des lacunes actuels des sciences sociales anglo-saxonnes ${ }^{20}$. En réalité, une nouvelle lecture de Weber confirme qu'il pensait qu'« il s'agissait d'États nationaux en compétition dans un climat de conflits et de guerres perpétuel (...). De cette alliance dictée par la nécessité entre État et capital naquit la classe citoyenne nationale, la bourgeoisie dans le sens moderne du terme. Ainsi, c'est le système d'État national fermé qui a permis au capitalisme de se développer $»^{21}$. Selon Tom Nairn, « au fur et à mesure que le capitalisme s'est développé et a écrasé les anciennes formations sociales, celles-ci avaient toujours tendance à tomber en même temps que leurs défauts internes. Ces points faibles, il s'agit là d'une vérité des plus élémentaires, avaient presque toujours trait à la nationalité (dans certains cas biens connus, des conflits religieux enracinés pouvaient conduire au même résultat) $»^{22}$. Des interprétations récentes cherchent à " ramener l'État sur la scène » en tant que principal facteur de changement et de développement économique, social et politique $^{23}$. Une fois de plus, l'intervention de l'État est considérée comme essentielle pour les pays connaissant un retard économique. Elle est adaptable, non seulement aux pays du Tiers-Monde "en voie de développement" et aux ex-colonies, mais aussi aux sociétés post-communistes.

Les sociétés qui présentent un décalage de compétence sont facilement écartées du circuit. Elles sont marginalisées ou mises à la périphérie. Si elles succombent à la pression de la marginalisation, elles doivent alors subir une désintégration de leurs modes de vie traditionnels - c'est-à-dire une régression sociale -, auquel cas elles sont tout simplement opprimées; sinon, elles sont converties en satellites dépendants des sociétés plus développées (...). Dans ce cas, du point de vue de l'économie dominante, des "économies esclaves" font leur apparition ${ }^{24}$. plus haut, dont le choix est essentiellement idéologique et politique. Les élites postcommunistes devront faire un inventaire des moyens mis à leur disposition afin de 
choisir le modèle de développement, la voie à suivre et la vitesse de changement, tout en sachant que leurs sociétés devront supporter le coût de ces réformes, se préparant à faire face aux conséquences politiques. Dans nos ethnocraties où la démocratie n'est, dans un futur d'après-guerre incertain, ni plus ni moins qu'un projet, les cliques dirigeantes peuvent être tentés d'user de leur monopole de coercition pour imposer des modèles de développement économiques autarciques et protectionnistes "de façon temporaire" aux sociétés civiles, ainsi que F. List l'a proposé.

31 L'autarcie économique est généralement une dimension de l'idéologie nationaliste. Les chauvinistes militants du type de Jirinovski et les communistes conservateurs s'insurgent déjà contre «la liquidation du pays et du patrimoine national». En Azerbaïdjan et en Croatie, les nouveaux États ont retiré les compagnies pétrolières nationales les plus lucratives du programme de privatisation. En Croatie et en Serbie, l'État a en fait nationalisé des compagnies anciennement sous contrôle nominal des ouvriers et dans lesquelles les employés s'étaient investis pendant des années. L'État a ensuite vendu ces entreprises à des personnes privées. Cela revenait à une expropriation de force ${ }^{27}$.

32 En outre, nos ethnocraties n'ont qu'un monopole relatif de la violence. Les milices de partis et les armées privées ont proliféré durant la guerre. En juin 1993, en Azerbaïdjan, le colonel Suret Huseinov se rebella et ses troupes réussirent à évincer le Président élu Elchebey. Lors d'un compromis avec le vétéran communiste Haydar Alyev, Huseinov s'imposa comme Premier ministre. Plus tard, Alyev, devenu entretemps Président, accusa Huseinov d'un nouveau complot et réussit à l'éliminer, mais c'est alors que s'ouvrit un nouveau défi, des unités spéciales du ministère de l'intérieur ayant appuyé Alyev. Auparavant, des irréguliers azerbaïdjanais qui portant les insignes des Loups gris turcs avaient déclaré leur propre guerre contre les milices du parti dashnak arménien. La milice croate fasciste HOS constituait, avant son intégration dans l'armée régulière HVO (Conseil de défense croate), les troupes de choc croates en Bosnie-Herzégovine. En août 1994, les "forces de défense territoriale" de la soi-disante Bosnie occidentale autonome de Fikret Abdić furent impitoyablement écrasées par les troupes régulières des Musulmans bosniaques, mais elles contre-attaquèrent en alliance avec les forces irrégulières serbes. Les "Bérêts rouges" serbes du capitaine Dragan, les "Tigres" d'Arkan, les "Aigles blancs" et d'autres irréguliers et guerriers du week-end furent systématiquement engagés dans des opérations de "nettoyage ethnique" en Croatie et en Bosnie-Herzégovine. Pour les éléments sociaux déclassés recrutés pour de telles unités irrégulières, le butin, drappé de slogans patriotiques, est la seule motivation, et la guerre devient "un mode de production" spécifique.

\section{L'État-nation}

«Les frontières présumées de la nation sont les mythes et les souvenirs du groupe ethnique dominant. $»^{28}$ Aussi bien les frontières culturelles que les frontières territoriales des États-nations sont des constructions sociales et seules l'action et la conviction humaines leur donnent un sens ${ }^{29}$. Ces cartes mentales peuvent changer au cours du temps mais, en fait, elles constituent toujours la matrice complexe des conventions humaines. Les mythes de l'origine, de l'histoire, des souvenirs, de la culture, du territoire, des intérêts de l'État ou de la nation sont toujours réinventés par 
les élites dirigeantes qui cherchent à gagner ou à se maintenir au pouvoir au nom d'une population collective définie selon des critères ethno-nationaux.

Selon July Mostov, dont je partage les opinions, la lutte pour établir des ethnocraties implique cinq processus étroitement liés les uns aux autres :

- le changement de frontières, c'est-à-dire la création ou un nouveau tracé des frontières territoriales et symboliques, des frontières entre les différentes communautés et des frontières entre individus ;

- "l'édification nationale" dans laquelle la nation est "récupérée" sous sa forme originelle, avec son idéologie nationale, ses mythes, ses points de vue, sa "façon d'être" ; les liens primordiaux de la nation au passé sont reconstruits et exhaltés, donnant aux liens de sang mythiques une place centrale dans l'identité nationale ;

- "l'édification étatique" dans laquelle les institutions politiques et culturelles sont établies afin d'assurer la domination de la nation "recouvrée", de redéfinir le critère de citoyenneté et les porteurs des droits politiques ;

- le remplacement d'un sujet collectif par un autre, en réduisant le nombre des sujets politiques légitimes et en contrôlant l'accès aux arènes publiques ; et

- le changement des paysages - la destruction des villes et des marqueurs culturels et l'exclusion, l'expulsion et l'exode ${ }^{30}$.

Le problème est que de tels programmes politiques conduisent inévitablement à la confrontation avec les exclus et les expulsés qui, dans un réflexe de défense, se réunissent souvent autour de lignes nationalistes similaires. Le nationalisme contre le nationalisme, la violence contre la violence et les guerres pour la reconquête de ces cartes mentales, ces frontières et territoires "nationaux" imaginés deviennent le nouvel ordre du jour. Les églises et mosquées appartenant à l'“ennemi" n'ont pas systématiquement sauté par hasard, ni en Croatie ni en Bosnie-Herzégovine, ni, non plus en Azerbaïdjan ou en Arménie. Ce n'est pas non plus sans intention que des villes entières symbolisant une "culture étrangère" ont été détruites. Quand la cité médiévale de Dubrovnik a été bombardée par l'artillerie de l'armée yougoslave, Vučurević, un des seigneurs de guerre serbe, a déclaré, comme on pouvait s'y attendre, qu'après sa «libération (...), nous [Serbes] construirons une Dubrovnik nouvelle et plus belle». Quand le général du HVO, Praljak, a visé le magnifique pont aux arches élyptiques du XVI ${ }^{\text {ème }}$ siècle au dessus de la rivière Neretva à Mostar, il détruisait un des symboles les plus importants de la culture musulmane bosniaque. Dans le tout nouvel espace ethnique, les noms des villes et des rues sont modifiés pour imposer une nouvelle géographie politique. Les arguments utilisés sont "historiques" («c'est la terre de nos ancêtres!»), ou démographiques (n'importe quelle statistique prouvant qu'à une certaine époque "nous formions la majorité »), et, lorsque l'histoire et les statistiques ne sont d'aucun recours, alors des raisons géostratégiques sont invoquées (ligne de défense naturelle, communication entre deux zones, ou tout simplement lebensraum). En glorifiant les rois, poètes, artistes et héros "nationaux" connus ou anonymes, les nouveaux repères tentent d'établir un lien spécial entre la nouvelle communauté et le passé réinventé. Le programme de la Grande Serbie contre la Grande Albanie, de la Grande Serbie contre la Grande Croatie, de la Grande Arménie contre le Grand Azerbaïdjan, de la Grande Géorgie contre la Grande Abkhazie - ennemis jumeaux, chacun d'entre eux avec sa propre projection exclusive d'un "espace national". Ironiquement, des ennemis jumeaux acharnés peuvent trouver éventuellement plus pratique de coopérer dans la diabolisation et la destruction d'un troisième frère, 
comme c'est le cas en Bosnie-Herzégovine. Une fois le nouveau but "moral" et territorial atteint, le combat peut s'étendre à la famille entière.

Deux principes internationalement reconnus facilitent considérablement ces processus : le droit à l'autodétermination nationale et la priorité des droits collectifs sur les droits individuels. La soi-disant communauté internationale n'a pas su faire la distinction entre ces principes basés sur l'intolérance, la discrimination et le principe démocratique de l'autodétermination populaire et de l'égalité citoyenne, du moins dans la tragédie bosniaque ${ }^{31}$. En choisissant, dès le départ, de parler seulement aux représentants des trois partis nationalistes bosniaques (musulman, serbe et croate) et en excluant tous les autres ( $26 \%$ d'abstention aux premières élections parlementaires au cours desquelles un pourcentage égal a voté pour de plus petits partis d'origine communiste), les médiateurs internationaux rendirent légitimes les seigneurs de la guerre actuelle et, de facto, acceptèrent leur logique perverse de retraçage de cartes. Bien pire, ils fermèrent les yeux sur l'aggression et les conquêtes territoriales de la Grande Serbie, ainsi que la "bantoustanisation" de la Bosnie-Herzégovine, et ils encouragèrent indirectement la Grande Croatie dans ses ambitions d'annexer des parties du territoire bosniaque.

De même, la communauté internationale n'a rien fait contre les politique de "purification ethnique" dans le Caucase et a été plutôt clémente quant à l'invasion des territoires voisins par les Grands Arméniens en Azerbaïdjan. De toute évidence, la diaspora arménienne en Russie, aux États-Unis d'Amérique et en France a une influence considérable.

La description d'Ernest Gellner du processus historique de l'émergence d'une nation ruritanienne imaginaire se séparant de l'empire de la Mégalomanie, pour finalement proclamer son indépendace bien méritée, est paradigmatique ${ }^{32}$. C'est ainsi que sont nés les États-nations à travers l'Europe centrale et orientale ; ainsi que de la décomposition de l'Empire russe / soviétique. Les nations sont aussi des constructions sociales. Gellner insiste: "c'est un mythe que de considérer les nations comme un moyen naturel, donné par Dieu, de classer les hommes (...) ; le nationalisme se saisit parfois des cultures préexistentes et les transforme en nation, parfois les invente, souvent oblitère les cultures préexistentes: telle est la réalité » ${ }^{33}$. Il déclare qu'une "population homogène" est un besoin de la société industrielle, c'est-à-dire que c'est la classe ouvrière lisant et écrivant la même langue et partageant les mêmes valeurs qui donne naissance à la nation. La nation résulte de réalités matérielles objectives, il en va de même pour les efforts du peuple à établir une nationalité. Avec l'industrialisation, « les populations illéttrées, à demi affamées, qui ont quitté en masse leur campagne et leur ghetto culturel dépassé pour se déverser dans ces creusets que sont les bidonvilles, désirent ardemment l'intégration à une zone culturelle qui a, ou semble pouvoir acquérir, un État qui lui soit propre et qui promette de donner, ultérieurement, une totale citoyenneté culturelle, (...), un emploi, etc. $»^{34}$.

Quant à savoir comment les manœuvres ethniques fonctionnent en pratique, il vaut mieux se référer au laboratoire vivant d'Asie centrale où les populations préindustrielles furent mélangées, séparées, importées et déportées, manipulées, sédentarisées et corrélées dans "leurs" Républiques et territoires autonomes. Des écritures et des langues standards (changeantes) furent établies et des institutions culturelles "nationales" et des plans quinquennaux de développement furent créés. Bien que différentes identités coexistent et que le processus d'“édification nationale" 
ne soit pas encore achevé, ces exemples prouvent que la violence de l'État et la modernisation peuvent effectivement modeler une conscience fragmentée tribale et clanique en une identité nationale plus ou moins unificatrice ${ }^{35}$. En Asie centrale, le communisme a inventé et créé - ou, si on préfère, cyniquement manipulé - les nationalités. Contrairement à l'idée reçue, il ne les a pas "réprimées" ${ }^{36}$. Dans le Caucase, c'est l'État soviétique qui a finalement permis à la nation arménienne décimée et éparpillée de survivre, en lui fournissant un lieu de regroupement dans sa république montagneuse titulaire. D'un autre côté, il est vrai que Staline a déporté et "puni" collectivement des groupes nationaux entiers du nord-Caucase, de Crimée et de la région de la Volga. En effet, c'est à cause de leur ethno-nationalité que ces groupes souffrirent de la répression ${ }^{37}$.

40 C'est dans la Yougoslavie fédérale communiste que les Slaves macédoniens ont eu, pour la première fois dans leur histoire, leur entité étatique autonome, des institutions culturelles et une langue à part. Et c'est également dans ce pays que les Slaves musulmans de Bosnie-Herzégovine ont été reconnus pour la première fois comme une "nation" à part entière, distincte des Serbes et des Croates. Les Albanais du Kosovo forment la seule nationalité qui puisse se plaindre d'une quelconque répression, mais elle a aussi profité d'une certaine autonomie pendant environ une dizaine d'années.

41 Mais les nations ne sont pas uniquement créées d'en haut par la manipulation des élites et la coercition de l'État. Miroslav Hroch nous rappelle pertinemment que les nations émergent effectivement "d'en bas" au cours d'un développement historique long et compliqué. Il définit la nation comme " un large groupe social intégré, non par un, mais par toute une combinaison de liens objectifs différents - économiques, politiques, linguistiques, culturels, religieux, géographiques, historiques -, et leur projection subjective sur la conscience collective $\aleph^{38}$. Beaucoup de ces liens peuvent se substituer les uns aux autres - certains jouant un rôle particulièrement important dans l'étape de construction de la nation, mais trois d'entre eux se révèlent irremplaçables: (1) une "mémoire" ou un "mythe" d'un certain passé commun considéré comme la "destiné" du groupe entier ou, du moins, de ses constituants de base; (2) la densité des empreintes culturelles ou linguistiques permettant un niveau de communication sociale plus élevé tant au sein du groupe qu'à l'extérieur; (3) une conception de l'égalité formelle de tous les membres du groupe en tant que société civile. D'autre part, il établit une distinction claire entre l'identité nationale collective, le mouvement national et l'idéologie nationaliste. Dans son approche, le nationalisme n'est qu'une forme de conscience nationale parmi tant d'autres, ou pour être plus précis, "cette perspective qui donne une priorité absolue aux valeurs de la nation par rapport aux autres valeurs et intérêts ».

Les buts du mouvement national couvrent trois groupes de demandes: (1) le développement d'une culture nationale fondée sur la langue locale et son emploi dans l'éducation, l'administration et la vie économique; (2) l'accomplissement des droits civils et de l'auto-administration politique, tout d'abord sous forme d'autonomie et finalement sous forme d'indépendance ; (3) la création d'une structure sociale complète à partir du groupe ethnique, comprenant des élites cultivées, une administration et une classe dotée d'un fort esprit d'entreprise, mais aussi, si nécessaire, des paysans libres et des ouvriers organisés. Chaque mouvement national qui réussit passe par trois phases consécutives : (A) une phase de sensibilisation nationale ; (B) une phase de mobilisation nationale ; et (C) une phase associée à la fondation de l'État-nation. C'est seulement 
lorsque les buts cités ci-dessus sont réalisés que le mouvement se différencie en branches démocratique, libérale ou conservatrice-cléricale ${ }^{39}$. Dans nos pays, c'est un procédé qui dure toujours. Tous les programmes politiques sont identiques et il est pratiquement impossible de faire la différence entre la gauche et la droite à cause du chauvinisme et de l'autoritarisme particulièrement développés au sein des différents partis. En fait, la droite nationaliste extrémiste et la gauche conservatrice d'origine communiste se retrouvent souvent du même côté lors des débats parlementaires. La coopération entre le parti socialiste de Serbie de Milošević (l'ancienne Ligue communiste de Serbie) et le parti radical serbe de Vojislav Šešelj (monarcho-fasciste) n'est pas un exemple isolé. L'électorat lui-même ne suit pas les programmes des partis, mais des leaders isolés, tous du type caudillo.

L'ethnicité, ou le mythe des origines communes et des liens de sang, n'est pas identique au concept de nationalité, bien qu'elle fut souvent intégrée dans le processus d'édification de la nation, particulièrement en Europe de l'est. «L'identité ethnique est une des nombreuses identités dont les gens puissent disposer. Elle est développée, exposée, manipulée, ou ignorée selon les demandes de situations particulières (...). Au niveau de l'individu, l'identité ethnique est aussi bien un état mental qu'une stratégie possible (...). Dans la pratique, l'identité ethnique est un trait acquis et utilisé de l'identité humaine, sujet aux arrangements, à la mise à l'écart, aux manipulations et à l'exploitation. $»^{40}$ Dans nos régions, le nationalisme en tant qu'idéologie politique a exploité et exploite toujours avec succès l'ethnicité. «En fait, l'ethnicité pénètre la sphère politique en tant que "nationalisme" quand la base économique de la vie sociale exige l'homogénéité ou la continuité culturelle (et non pas l'absence de classe) et que, par conséquent, les différences de classe liées à la culture deviennent nocives alors que les différences de classe discrètes et insensibles, du point de vue ethnique, demeurent supportables $»^{41}$. La preuve est faite que la phase la plus violente dans les conflits ethno-nationaux est liée à une industrialisation précoce.

Tandis que la vague de modernisation déferle sur le monde, il est certain que chacun ou presque, à un moment ou à un autre, a une raison de ressentir un traitement injuste et qu'il peut identifier les coupables comme appartenant à une autre "nation". Quand on peut également identifier un nombre important de victimes comme étant membres de la même nation que la sienne, alors naît un nationalisme. S'il triomphe, et ce n'est pas le cas pour tous les nationalismes, alors naît une nation. ${ }^{42}$

Le nationalisme et l'identité nationale ne sont pas non plus synonymes. Il est important de faire la distinction entre les idéologies politiques nationalistes et l'autoidentification nationale qui comporte des caractéristiques culturelles et légales. L'individu peut s'identifier culturellement en tant que Croate, Serbe, ou Arménien, Tchétchène, ou Abkhaze, simplement parce qu'il (ou elle) est né(e) ou a évolué dans une communauté donnée sans être nationaliste ou sans «donner une priorité absolue aux valeurs (supposées) de la nation ». Il s'agit d'une identité parmi d'autres dont peut disposer l'individu. Ces identités peuvent même se compléter les unes les autres, à l'exemple de cette déclaration, assez commune dans l'ancienne Yougoslavie: «Je suis Musulman bosniaque de nationalité, mais je suis athée et citoyen yougoslave». La théorie des identités gigognes de R. Taras n'est pas dénuée de sens ${ }^{43}$. Utilisant une autre perspective et partant de l'individualisme ethique, Yeal Tamir proposa le terme quelque peu malencontreux de "nationalisme libéral" qui demeure culturellement nonexclusif, politiquement pluraliste et respectueux du droit des autres individus à être 
différents. Selon elle, ce "nationalisme" large d'esprit est associé à la démocratie libérale et accroît la liberté individuelle et l'éventail des choix rationnels ${ }^{44}$. Contrairement aux idéologies nationalistes xénophobes, l'auto-identification nationale libérale ne souffre pas de complexe d'infériorité ou de supériorité, qui sont en fait les deux revers de la même médaille. Il n'est pas culturellement autarcique et exclusif, ni politiquement agressif envers les autres identités avec lesquelles il coexiste et cohabite. C'est juste un genre d'adresse personnelle que l'on porte, dans un monde subdivisé en États-nations, où tout le monde a sa propre étiquette.

Les mouvements nationaux sont comme les mouvements sociaux analysés par Touraine. Leur dimension ethno-nationale peut être exagérée pendant la phase B de mobilisation politique décrite par Hroch, comme ce fut le cas dans les Balkans et dans le Caucase. Une fois qu'ils ont atteint leurs buts - la naissance de l'État-nation -, ils ont tendance à se différencier politiquement et à se fragmenter en plusieurs partis et groupes différents souvent opposés. Bien qu'il soit lent et souvent obscurci par des luttes pour le pouvoir entre d'éventuelles cliques dirigeantes, un tel processus démarre immédiatement après la déclaration d'indépendance politique.

Aussi paradoxal que cela puisse paraître au néophyte, les arguments théoriques fondamentaux avancés pour l'autodétermination et la libération nationales trouvent leurs racines dans la littérature marxiste. Prévoyant la décomposition de l'AutricheHongrie, Karl Kautsky écrivait en 1887 : «À mesure que les antagonismes économiques s'aiguisent, que chaque région économique essaie de développer son industrie urbaine ou rurale, mais peut de moins en moins le faire sans entraver le développement économique du voisin, les différentes régions économiques de l'Autriche tendent à se séparer et la politique de "réconciliation" des nationalités devient plus difficile »" Je peux aussi citer Lénine qui a écrit: "la formation d'États nationaux qui satisfont le mieux aux exigences du capitalisme moderne est donc une tendance propre à tout mouvement national (...); et pour toute l'Europe occidentale - bien mieux, pour le monde civilisé tout entier - ce qui est typique, normal en période capitaliste, c'est donc $l^{\prime}$ Etat national $»^{46}$.

Dans la même lignée de pensée, un universitaire expliquant les raisons de la décomposition de l'URSS et de la Yougoslavie remarquait que

comme le marché, la production de produits de base et l'accumulation du capital avaient conquis le monde, de même, l'État national avait pris place en tant que forme archétype du pouvoir politique organisé (...). L'idéal de l'État national était devenu partie intégrante de l'idéologie dominante à travers le système mondial, à tel point que des mouvements, différents sur bien des points de ceux que [Lénine] avait connus, avaient repris cet idéal (...). Du fait de la place centrale de la nationalité dans l'idéologie du système [mondial], la réaction de la population d'un État national donné face à la crise économique et politique était de chercher à créer une nouvelle nation, basée sur des critères différents de l'ancienne. ${ }^{47}$

De toute évidence, des systèmes aussi complexes que ceux de l'Union soviétique et la Yougoslavie évoluent.

Les rôles de leurs parties se multiplient et deviennent plus puissants, aux dépends $\mathrm{du}$ centre. Dans un système décentralisé, où les unités territoriales ont des responsabilités de plus en plus importantes pour gérer leur énergie, l'intervention entre les composants conduit à l'autogestion. Cette interaction peut être compétitive ou coopérative, mais elle est pratiquement toujours houleuse. Sans conflit, il n'y a pas de changement, et sans changement, il n'y a pas de progrès. La 
manifestation la plus importante d'un tel changement est la réorientation et le réalignement des unités territoriales politiques. ${ }^{48}$

\section{L'économie politique des relations internationales}

Toutes les idéologies nationalistes sont irrationnelles et, lorsqu'elles dominent l'État et la société, comme dans le cas de nos frères-ennemis, elles provoquent des effets désastreux. Elles sont destructrices et auto-destructrices. Du point de vue de l'économie politique et des relations internationales contemporaines, la structure même de l'État-nation moderne est aussi rationnelle que n'importe quelle autre structure humaine. Sa "rationalité" provient du système mondial, et non de l'État isolé. De ce point de vue, et tant que cette unité-État est intégrée dans le système mondial et joue son rôle dans la division internationale du travail, il ne s'agit pas de savoir quoi et qui remplit sa fonction. La taille de l'unité-État est également hors de propos. Le Luxembourg, Andorre et San Marino se débrouillent remarquablement bien, sans parler de Singapour, ni de Hong-Kong. Hong-Kong est toujours une colonie de la couronne britannique; la preuve que même l'indépendance politique n'est pas une précondition indispensable au progrès économique ou à l'intégration dans le système mondial. D'un autre côté, du point de vue de l'individu vivant dans une telle unité-État, ou du point de vue d'une communauté plus importante (minorité ou majorité) du nouveau État-nation, la formation étatique précédente peut apparaître "meilleure". Pour ceux qui sont persécutés, nettoyés, exclus et violés, pour les réfugiés et les personnes déplacées, pour ceux qui ont perdu leur sentiment de sécurité, leur emploi et leur maison, c'était certainement mieux avant, mais tout dépend, finalement, de la vision personnelle des choses. Dans la même ligne de pensée, on peut dire que l'Union soviétique et la Yougoslavie valaient "mieux" que toutes ces guerres et ces problèmes actuels. Cela ne veut pas dire qu'elles étaient plus "rationnelles" du point de vue de l'économie mondiale. En fait, on pourrait même ajouter que leurs économies étatiques dirigées se sont effondrées et que les deux fédérations se sont décomposées parce qu'elles n'étaient pas assez rationnelles pour le système mondial.

L'économie internationale libérale ou le "système capitaliste mondial" et le socialisme réel n'étaient pas dans les meilleurs termes. Cependant, ils "coexistaient" et faisaient du commerce. Il s'agissait essentiellement d'une relation mercantile, bien que les États "socialistes" fussent désireux d'importer la technologie occidentale par le biais de projets communs directs et de partenariats avec des fonds étrangers. Les termes commerciaux étaient ceux du système mondial. Dans la teminologie de l'école de la dépendence, cela signifie qu'ils étaient "inégaux", que le noyau capitaliste exploitait la périphérie socialiste européenne à travers des échanges inégaux. En termes économiques, le "système socialiste mondial", en tant que tel ou en tant qu'alternative soviétique au système de relations économiques internationales existant, n'a jamais pris forme. Le COMECON n'est jamais devenu un contre-poids à la Communauté européenne. Les tentatives individuelles les plus ambitieuses pour "briser les liens" avec le système capitaliste mondial, les "grands bonds en avant" chinois et la vivisection sociale des Khmers rouges ont tous deux finis en catrastrophe économique et humaine colossales. Depuis le tout début, le soi-disant système socialiste mondial, c'est-à-dire le regroupement des économies nationales "socialistes" dirigées, mutuellement non-intégrées, était, et resta jusqu'à son effondrement, un appendice sous-développé du système mondial. 
51 Bien sûr, le système mondial tira profit de cette relation inégale, mais le caractère des économies socialistes maintenant défuntes était tel qu'il ne permit pas la maximisation des profits comme c'est le cas actuellement pour l'expérience chinoise de l'économie de marché. Le système mondial peut s'accomoder de l'existence d'un secteur public en tant que tel, et d'une bureaucratie étatique, et même du monopole politique des élites communistes dans les pays périphériques. Les exemples de la Corée du sud, avec son imposant secteur public dirigé par des officiers militaires, et de la République populaire de Chine, le prouvent bien. L'absence d'un marché libre de marchandises, de main d'oeuvre et de capital et les obstacles administratifs imposés par l'État à l'égard de la libre circulation du capital à travers les frontières nationales créent des problèmes que le système mondial cherche à éliminer. Il importe peu que des unités étatiques disparaissent ou apparaissent au cours de développements politiques. Ce qui est important, c'est que le système s'étende et fonctionne avec le maximum d'efficacité et de profit. Au-delà du "conflit idéologique" camouflant des intérêts d'État économiques plus importants, on comprend pourquoi l'effondrement du socialisme a été salué avec enthousiasme par les libéraux.

52 Toutefois, le "système capitaliste mondial" n'est pas le monolithe que ses adversaires idéologiques aiment à croire. Ce n'est pas non plus le deus ex-machina des partisans occidentaux de l'économie de marché et de la démocratie. En fait, le système mondial de libéralisation du marché, des monnaies stables et de l'interdépendance accrue a connu de grands problèmes depuis la deuxième moitié des années 1970. Finalement, l'effondrement du socialisme réel ne fut pas un tel bienfait pour le système. Les quatre dernières années ont déjà démontré que le morceau était trop gros à avaler en entier. Il serait hors de propos dans cet essai de discuter les théories de dépendance et leur élaboration académique, mais, si l'on veut expliquer les défis actuels lancés à l'Étatnation, sa "souveraineté" et son "indépendance", nous devons analyser les relations entre l'État, le marché et le système mondial. Dès le début, nous devrions reconnaître qu'il y a des tensions entre l'économie mondiale fondée sur les principes du marché international et les économies domestiques basées sur l'intervention de l'État. Dans l'ébauche ci-dessous du système mondial et de ses problèmes actuels, qui est un thème d'une importance cruciale certaine pour tous les États-nations post-communistes, je m'appuierai sur des interprétations plus pragmatiques de l'économie politique internationale contemporaine, comme les analyses de Schumpeter ${ }^{49}$ ou les études plus récentes de Robert Gilpin ${ }^{50}$.

53 Pour résumer, l'économie internationale qui fit son apparition après la Seconde Guerre mondiale était une économie libérale fondée sur l'hégémonie militaire et économique nord-américaine dans un système hiérarchique d'États-nations. Comme Gilpin le souligne, cette prééminence américaine ne fut jamais défiée ; cela était dû en partie à la destruction des autres économies industrielles pendant la guerre.

Les liens de sécurité et les liens politiques entre les États-Unis d'Amérique et ses principaux alliés japonais et occidentaux fournit la structure politique nécessaire au bon fonctionnement de l'économie de marché libérale mondiale (...). Le leadership nord-américain et le système d'alliance créa une base stable et sûre pour le développement des relations économiques mondiales. Pour la première fois au monde, toutes les économies capitalistes étaient des alliés politiques. Les initiatives commerciales nord-américaines menèrent aux «rounds" successifs de libéralisation des tarifs. Le dollar servit de base au système monétaire international (...). Les États-Unis d'Amérique s'assurèrent du fardeau de la défense des démocraties industrielles, permettant ainsi aux Européens de l'Ouest et, surtout, 
aux Japonais de concentrer leur énergie et leurs ressources sur le développement économique. L'hégémonie nord-américaine apporta ce climat favorable dans lequel s'épanouissent les forces de l'offre et de la demande. Ainsi naquirent cette ère de croissance sans précédent et cette économie internationale en expansion continuelle..$^{51}$ la guerre du Viet-Nam et celui-ci devint évident avec la chute du système monétaire de Bretton-Woods et la crise pétrolière dans les années 1970. La part nord-américaine dans la production mondiale déclina rapidement, la productivité des États-Unis d'Amérique s'effondra petit à petit et l'auto-suffisance en matières premières diminua. Cependant, les États-Unis d'Amérique conservèrent leur domination économique et militaire, pouvoir militaire financé par un déficit budgétaire de plus en plus important, qui atteint plus de 200 millions de dollars chaque année. Par la réduction des impôts, l'administration Reagan a réussi à relancer temporairement l'économie, mais l'effet indésirable fut l'accumulation effrénée des dettes étrangères, publiques et privées qui doublèrent presque entre 1980 et 1985, passant de 4,3 à 8,2 milliards de dollars ${ }^{52}$. En fait, les États-Unis d'Amérique finançaient leur développement militaire et le maintien de leur hégémonie mondiale, principalement au travers de l'emprunt étranger, spécialement grâce à l'assistance financière fournie aux Japonais, aux Allemands et à certains pays arabes exportateurs de pétrole. Pour rembourser la dette extérieure, les États-Unis d'Amérique auront besoin d'un surplus d'environ 100 milliards de dollars par an, et ce jusqu'à la moitié du siècle prochain.

Pour conclure, "le déclin relatif de l'hégémonie américaine a sévèrement ébranlé la structure politique stable qui avait maintenu l'expansion de l'économie libérale mondiale dans l'ère de l'après-guerre, et, de fait, le protectionnisme grandissant, l'instabilité monétaire et la crise économique se sont développés ${ }^{53}$. En même temps, l'exploitation de leur position économique dominante par les nord-Américains déplaît de plus en plus à ses partenaires économiques, à savoir les Européens et les Japonais, qui sont eux-mêmes incapables, ou qui n'ont guère l'intention, d'assumer davantage de responsabilité dans la gestion du système global et qui poursuivent leurs propres objectifs limités. Les intérêts paroissiaux des différents pays européens, renforcés par la compétition politique interne et une longue récession économique, repoussèrent le processus d'intégration d'Europe de l'ouest au siècle prochain. Désunis, ils durent accepter les demandes nord-américaines pour la libéralisation de leur politique agricole et l'abandon des barrières protectionnistes artificielles qu'ils avaient établies. En outre, le Japon est ébranlé par une crise politique interne, et est incapable de satisfaire, pour l'instant, les capitulations économiques extravagantes des nordsAméricains. Bien sûr, une telle situation affecte l'économie mondiale en général et, plus spécialement, la position des pays qui ne sont pas encore entièrement intégrés dans le système.

Il serait faux de croire que les pays post-communistes et ceux en voie de développement ne veulent pas s'intégrer, car chacun d'entre eux pourrait bien finalement être contraint de s'engager dans cette direction. En fait, le problème réside dans le fait que le système mondial fluctuant contemporain est incapable de tous les absorber d'un seul coup. On peut imaginer que des "eurocrates" et d'autres groupes d'experts sont en train d'élaborer des listes d'attente et de dates pour l'intégration graduelle des États-nations dans l'Union européenne, mais l'économie elle-même, particulièrement le capital transnational, procède par secteur. Dans l'ensemble de 
l'Eurasie, une compétition féroce s'est établie pour obtenir les meilleures positions dans les secteurs économiques les plus importants stratégiquement et lucrativement. En Europe de l'est et dans les pays successeurs de l'URSS, l'accent est mis, pour l'instant, sur le système bancaire et les moyens de financement où l'on s'attend à des bénéfices immédiats, ainsi que sur le pétrole et le gaz dont les gisements pourraient bien être plus importants qu'au Moyen-Orient. Enfin, dans une étape finale, similaire à la compétition pour le marché chinois, la dislocation de certaines industries commencera. Dans le processus, les élites dirigeantes locales seront courtisées, harcelées, corrompues et achetées. Mais, plus significativement, de nombreuses compagnies chercheront l'appui et la protection de leurs propres États et / ou entreront dans des alliances / cartels avec d'autres compagnies, se répartissant les marchés et éliminant les rivaux. C'est ce que Vinold Aggarval appelle le "protectionnisme libéral" ${ }^{54}$. Son poids général dans le système mondial pourrait bien devenir plus important, réduisant ainsi l'espace de manoeuvre indépendant et la souveraineté économique d'unités-États plus faibles et moins développées. Par ailleurs, il y a une tendance parallèle à la régionalisation de l'économie mondiale autour des trois pôles de développement économiques les plus importants, à savoir l'Amérique du Nord, l'Europe de l'ouest et la région Japon-Pacifique. Si cette tendance persiste, le reste du monde sera subdivisé en réserves marchandes dépendantes de ces trois pôles. La première tendance pourrait mener à des guerres commerciales entre États et la seconde à des conflits entre conglomérats économiques. Si mon interprétation de la dernière dévaluation du dollar américain est juste, alors il ne s'agissait pas simplement d'augmenter temporairement les exportations nord-américaines, mais aussi de ramener les économies d'exportation allemande et japonaise, en expansion, à l'obéissance. La "faiblesse" actuelle du dollar n'est certainement pas un handicap mais une preuve de la vigueur retrouvée de l'économie nord-américaine, dépendant seulement en partie des exportations. En effet, il semble qu'une fois de plus, les nordAméricains produiraient "plus, mieux et moins cher" que leurs rivaux. Un dollar "bon marché" pourrait finalement faire oublier la dette américaine et museler à nouveau le yen et le mark.

57 Nous vivons en ce moment une période de déséquilibre économique transitoire, où la position dominante des États-Unis d'Amérique est défiée à la fois par son propre développement intérieur et des facteurs extérieurs; mais, actuellement, aucune alternative à leur hégémonie ne se profile à l'horizon. Bien sûr, nous pourrions spéculer sur un monde "trilatéral" ou sur une ère post-hégémonique. Mais cela n'aiderait pas beaucoup nos États-nations nouveaux et faibles. Pour l'instant, ils représentent encore des territoires marginaux, mal intégrés dans l'économie mondiale. Ce sont des réserves où la saison de chasse vient de commencer. Cependant, cela ne veut pas dire que les élites dirigeantes des États petits et faibles soient complètement sans ressource. Si elles sont en mesure de comprendre le fonctionnement de l'économie politique des relations internationales et la nature de ses contradictions internes, alors, peut-être, peuvent-elles choisir la meilleure option et même préparer l'intégration la plus avantageuse de leur pays dans le système mondial. Malheureusement, nos ethnocraties, avec leur mentalité "ethno-nationaliste" très fermée, ne sont pas sur la même ligne de pensée et ne permettent pas à ces pays d'agir de manière constructive, ceux-ci étant mentalement incapables de saisir les chances qui se présentent. 


\section{Réalignement régional et réadaptation globale}

\section{illusions prématurées quant à l'avènement des marchés capitalistes, la démocratie} libérale et le monopole incontesté nord-américain du leadership mondial ${ }^{55}$. L'opinion publique est horrifiée devant les images télévisées de nos jeux barbares où l'on ne sait plus exactement qui tue qui, pourquoi, ni où, alors que tout est résumé dans l'étiquette déroutante de "conflit ethnique": Abkhazes contre Géorgiens, Géorgiens contre Ossètes, Tchétchènes contre Ingouches, Ingouches contre Ossètes, Tchétchènes contre Russes, Géorgiens contre Géorgiens, puis Arméniens et Azéris entre eux, Serbes contre Albanais, Croates contre Musulmans, Musulmans contre Musulmans, la minorité tutsi contre les Hutus au Rwanda et l'inverse au Burundi, des tribus contre d'autres tribus avec des noms et des appellations géographiques étranges. Effectivement, tout cela prête bien à confusion; d'ailleurs, on dirait que tous les protagonistes, criminels et victimes, surgissent d'une autre époque oubliée. qu'on y joue, se déroule désormais dans un théâtre qui nous est tout à fait inconnu, sur une scène qu'on ne reconnaît pas et au milieu de changements de décors imprévus, inattendus et que l'on comprend mal $\aleph^{56}$. L'ordre bi-polaire de la guerre froide, donna finalement naissance à un "nouveau désordre mondial" déjà rationalisé par les théories du chaos pour lesquelles le désordre est normal, inéluctable et contagieux ${ }^{57}$. Bien sûr, on peut envisager toutes sortes d'explications plus plausibles; dans les paragraphes suivants, je ferai un bref inventaire des considérations élémentaires d'espace et des configurations de force toujours changeantes. eurasien, lui-même composant et partie pivot du système des relations internationales, dans lequel les régions balkaniques et caucasiennes ont joué, et jouent toujours, des rôles géostratégiques particuliers et changeants. Avec leur "chaos ethnique", toutes deux peuvent être considérées comme des "laboratoires" internationaux spécifiques où les acteurs locaux, régionaux et extra-régionaux, les États-nations (ou, pour être plus précis, leurs élites politiques) se testent les uns les autres et testent leurs propres politiques toujours mal définies. Ils procèdent par expériences, petit à petit, partant à la reconnaissance du terrain et de ses potentialités, évaluant leurs propres intérêts et le poids des autres protagonistes. Dans ce processus, des cartes sont dessinées et testées. Les acteurs changent de rôles et d'alliés. Les bellicistes deviennent pacificateurs et vice versa. Les médiateurs internationaux voient leurs mandats renouvelés. Tous les éléments semblent en fluctuation.

\section{Le laboratoire de la Yougoslavie}

61 Durant la guerre froide, les Balkans étaient une zone à part, où un "équilibre hégémonique" était maintenu grâce à la formule 2 (Grèce et Turquie, bloc des pays de l'Ouest) + 2 (Bulgarie et Roumanie, bloc des pays de l'Est), + 2 (Yougoslavie et Albanie, pays en dehors des blocs). Après la défaite des communistes grecs dans la guerre civile, défaite évoquée en filigrane dans le traité de Yalta, mais vite oubliée, les Balkans furent transformés, sous l'égide des deux grandes puissances, en une région d'endiguement mutuel, dans laquelle la Yougoslavie non-alignée de Tito joua un rôle particulier. Le 
système régional d'endiguement fonctionna remarquablement bien, ceci en dépit de crises occasionnelles, jusqu'à ce que l'implosion de l'Union soviétique rouvre la boîte de Pandorre des guerres balkaniques.

Dans l'ensemble, personne en Occident ne se souciait réellement de la survie d'un Étattampon si grand (en fait, le plus grand et le plus peuplé de la région, si on fait exception de la Turquie asiatique). Pire encore, la survie de la Yougoslavie était liée au programme réformiste du Premier ministre, Ante Marković, et l'aider effectivement aurait de facto signifié donner une chance au communisme de marché en Europe centrale, formule qui pouvait s'imposer comme une alternative à l'effondrement total de l'économie dirigée. Ceci était hors de question, du moins pour le gouvernement de Bonn qui était encore dans l'euphorie de la réunification allemande et pour qui la Yougoslavie était également une occasion de montrer son autorité à ses partenaires européens. De même, le Vatican s'y opposa pour des raisons historiques et idéologiques évidentes, et les petits pays alentour étaient trop heureux de se débarasser de ce voisin présomptueux. Les Français, les nord-Américains hésitèrent pour d'autres raisons et les Britanniques mirent leur expérience colonialiste de traceurs de cartes à disposition. Contrairement aux théories de la conspiration, entretenues par de nombreux intellectuels balkaniques, ces puissants étrangers n'avaient pas de vision stratégique cohérente du réaménagement de l'espace yougoslave; tout était réduit à de petits remaniements en zig-zag décidés au jour le jour. Même si l'on savait que les "conflits ethniques" pouvaient prendre de l'ampleur, personne n'a vraiment essayé de les enrayer tant que c'était encore possible. Dans un premier temps, on laissa faire les Hutus et les Tutsis yougoslaves, et l'espace précédemment organisé fut tranformé, selon les termes des géostratèges, en une "zone d'éclatement".

Les Européens de l'ouest, les seuls à pouvoir canaliser le cours des événements, étaient désunis. C'était surtout parce qu'ils n'avaient pas décidé des futures frontières de leur Europe unie. N'étant pas préparés à l'effondrement du communisme, ils applaudirent avec enthousiasme la chute du rideau de fer et tout le monde à l'Est se mit à croire sérieusement à l'adhésion de leur pays à la communauté européenne tant idéalisée. Dans l'espoir d'une vie plus digne et meilleure, les populations pensèrent que le projet communiste était le principal obstacle les séparant d'une société d'abondance matérielle. Pour elles, une fois ces régimes effondrés, la question de l'intégration de leur pays dans la Communauté européenne ne serait qu'une simple formalité, à l'instar de la réunification allemande. La suite des événements prouva qu'elles avaient tort. L'Allemagne a toujours des problèmes pour digérer sa partie orientale, et l'Europe occidentale dans son ensemble semble dépassée par le fardeau des pays d'Europe de l'est. Le projet audacieux de l'intégration politique stipulé dans le traité de Maastricht est, de facto, repoussé à un avenir plus propice. Au mieux, quelques pays européens de l'est seront admis dans l'antichambre, mais ils devront prouver, au siècle prochain, qu'ils méritent la promotion. Finalement, les Américains, qui se réjouissaient du retard de l'unification européenne, offrirent leur partenariat pour la formule décorative de paix.

Dans un deuxième temps, l'Occident, en mal de nouvelles idées pour résoudre la crise yougoslave, estima qu'après tout, les frontières entre l'empire occidental et l'empire oriental romain n'étaient pas si mal que cela: finalement, on pourrait séparer la chrétienté (catholique et orthodoxe) en deux zones d'intérêts, créant ainsi deux entités-États qui se contrebalanceraient et se bloqueraient mutuellement: une 
confédération bosno-croate (contrôlant l'élément bosniaque musulman) et la Yougoslavie croupion (Serbie et Monténégro). De plus, chacune de ces entités serait régionalisée (autonomies régionales), apportant ainsi une solution aux minorités nationales : les Serbes en Croatie, les Albanais et même les Hongrois en Serbie.

La Macédoine, avec son importante minorité albanaise, suivra probablement le même schéma. Pour l'instant, elle joue le rôle d'État-tampon entre la Serbie, la Bulgarie, la Grèce et l'Albanie. Cependant, elle pourrait être transformée en État-charnière, en médiateur-clé dans le sud-est de l'ancien espace yougoslave. Le même rôle pourrait être attribué à la Slovénie dans le nord-ouest. Bien sûr, la Serbie, en tant qu'“État asymétrique" typique ${ }^{58}$ et principal fauteur de troubles dans les Balkans, devra être domptée. Le groupe de contact (États-Unis d'Amérique, Grande Bretagne, France, Allemagne, Russie) a, de toute évidence, opté pour la "bantoustanisation" de l'ancienne Yougoslavie. Dans l'esprit des organisateurs, cette solution avait pour but la pacification des ethnocraties, dont la direction de leurs entités territoriales respectives aurait été garantie, et devait aussi maintenir l'équilibre entre les différentes parties fragmentées. Il s'agit d'une formule de transition qui est censée garantir la trève et, à terme, ouvrir des canaux de communication entre les ennemis actuels. Les puissances impliquées espérèrent bénéficier d'un temps de répit afin de réaffirmer leurs intérêts et définir le rôle de leur région pacifiée et, éventuellement, intégrée économiquement dans la future "architecture" européenne.

L'idée générale est de transformer la zone d'éclatement yougoslave actuelle en une "région passage" entre le domaine de l'Europe occidentale (rattachant également la Grèce à l'Union européenne), l'Eurasie russe et le Moyen-Orient. Le domaine eurasien est, de toute évidence, dominé par la Russie, qui aura sans doute accès au passage des Balkans. C'est pourquoi, entre autres raisons, les nord-Américains l'ont cooptée, et c'est la raison pour laquelle ils ont donné aux élites dirigeantes russes l'impression qu'elles participaient à l'élaboration du nouvel ordre mondial ${ }^{59}$.

Bien que ce ne fut pas écrit dans ce scénario, il peut se trouver que l'administration Clinton ouvrit par inadvertance les portes de la Méditerranée aux Russes ${ }^{60}$. En commentant l'attitude nord-américaine face à la Russie post-communiste, Zbigniew Brzezinski parle de "politique d'optimisme idéaliste", conséquence directe de l'«amnésie historique» de Washington, et met en garde contre les impulsions impérialistes russes, les coalitions rouge-brun et le nationalisme russe, ainsi que contre le rôle grandissant de l'armée dans la prise de décision des priorités politiques ${ }^{61}$.

\section{Le laboratoire du Caucase}

Passons maintenant au laboratoire du Caucase où, bien avant l'effondrement de l'Union soviétique et l'émergence de nouveaux États-nations, des conflits sont apparus. Sur ce point, nous devons nous pencher plus particulièrement sur les pays à proximité de la Russie, où le rôle de celle-ci en tant que principal agent de paix eurasien est en train de se jouer. Bien sûr, dans cette région proche de la Russie, le Caucase n'est qu'un laboratoire parmi d'autres. Avec le laboratoire d'Asie centrale, il sépare la Russie du Moyen-Orient et du sous-continent indien. Ces deux sous-régions de l'Eurasie sont bien plus importantes pour la Russie que les nombreuses tentations qu'offrent les Balkans. De plus, l'issue de la lutte interne pour le pouvoir à Moscou, en parallèle avec la lutte entre le centre russe et ses régions périphériques et républiques autonomes, se jouera 
en grande partie dans ces régions proches. À ce niveau, les différentes factions politiques russes se font compétition et, peu à peu, Moscou rétablit sa prééminence.

Contrairement aux Balkans considérés comme un possible passage, le Caucase devrait probablement demeurer une zone-tampon classique, séparant l'actuel "trou noir" russe $^{62} \mathrm{du}$ Moyen-Orient. Pour la première fois dans l'histoire moderne, la Russie n'a pas de frontières communes avec le Moyen-Orient, région stratégique d'importance mondiale avec ses réserves de gaz et de pétrole, dominée depuis la guerre du Golfe par les intérêts nords-américains. Certes, il y a quelques États "récalcitrants" dans la région, mais l'Iran, l'Irak et le Soudan ne peuvent rien faire pour changer la configuration régionale générale de la stabilité hégémonique imposée par les ÉtatsUnis d'Amérique.

Il en va tout autrement au nord des frontières turques et iraniennes, où les Russes, bien que n'ayant jamais quitté les lieux, n'ont pas encore réalisé un retour spectaculaire. Alors qu'Eltsine était occupé par la lutte pour le pouvoir avec le Parlement central, des commandants militaires russes locaux, des attammans cosaques et d'autres mafias (avec certainement des relations importantes à Moscou) lancèrent leurs propres politiques dans le nord-Caucase et au Caucase. Des équipements militaires et des aides logistiques étaient à la disposition des Géorgiens, Abkhazes, Ossètes, Ingouches, et autres factions en guerre. Des mercenaires russes offrirent leurs services aux plus offrants, les pratiques de location de tanks, d'avions ou de batteries d'artillerie ${ }^{63}$, plus tard reprises en Bosnie, étaient plutôt une règle qu'une exception. Fort d'un tel appui, les séparatistes abkhazes et les "irréguliers" arméniens connurent un succès considérable et conquirent des territoires immédiatement ethniquement nettoyés.

71 Les témoignages du front abkhaze confirmèrent le fait que des mercenaires russes et des volontaires du Nord-Caucase anti-russes combattaient ensemble contre les Géorgiens. Autre coup de théâtre, le président géorgien évincé, Zviad Gamsakhourdia, ennemi supposé des montagnards du Nord-Caucase, reçut un accueil chaleureux à Grozny et le leader tchétchène Djokhar Doudaïev mit à sa disposition une aide logistique pour monter une offensive contre Tbilissi. Son retour fut empêché à la dernière minute par une intervention des troupes russes qui sortaient ainsi de leur "neutralité" et aidaient les loyalistes de Chevardnadzé en déroute. Après coup, Édouard Chevardnadzé ne put faire autrement que signer le traité de la CEI (Communauté des États Indépendants). Ainsi, la Géorgie, anciennement récalcitrante, dut rentrer au bercail.

Derrière ce chaos apparent, on peut reconnaître un certain modèle. Pas à pas, Moscou réussit à s'imposer à nouveau comme la puissance principale et médiateur le plus important de la région. L'Azerbaïdjan, qui entra puis se retira de la CEI, se repentit et rejoignit la structure communautaire dominée par les Russes, ne trouvant rien d'autre pour arrêter l'offensive arménienne. Auparavant, Moscou avait déployé ses soldats de la paix entre la Géorgie et sa région autonome d'Ossétie du sud, entre l'Ossétie du nord et l'Ingouchie naissante, entre l'Ossétie du nord et l'Ossétie du sud, dans la république autonome de Kabardino-Balkarie, entre la Tchétchénie et le Daghestan ${ }^{64}$. Cependant, la Tchétchénie séparatiste s'avéra un gros morceau à avaler. Après l'échec des troupes de Djokhar Doudaïev à ramener le pays au sein de la fédération russe ${ }^{65}$, Eltsine envoya ses troupes russes. L'expédition militaire, mal organisée, dégénéra en guerre sanglante. En bref, l'administration Eltsine, ayant survécu au défi du parlement central "rebelle" avec l'aide de l'armée et du soi-disant complexe militaro-industriel ${ }^{66}$, rétablit petit à petit 
l'autorité de Moscou au delà de la périphérie de l'ancien empire russe. Les régions et les républiques autonomes centrifuges de la fédération russe sont sur le point d'être sous contrôle central. Pour les intérêts géostratégiques de Moscou dans la région, le NordCaucase est particulièrement important, car c'est une porte sur le Caucase éclaté. La région doit être pacifiée et mise sous contrôle central.

Un parallèle intéressant pourrait être fait entre les interventions de l'ancienne JNA (Jugoslovenska Narodna Armija) en Slovénie et l'expédition russe en Tchétchénie. Comme pris dans le même scénario, ils exposèrent l'incompétence et la mollesse des structures de commandement militaire. Des jeunes recrues non formées furent envoyées au front et descendues comme des pigeons d'argile par les forces de défense locale. Dans le cas yougoslave, l'armée fut immédiatement retirée et les officiers "yougoslaves" remplacés par des officiers serbes pro-Milošević. Plus tard, pendant la guerre en Croatie et en Bosnie-Herzégovine, l'armée fut systématiquement ethniquement purgée et transformée en armée serbe. Il se pourrait que l'épisode tchétchène serve le même objectif et que l'armée russe soit maintenant désoviétisée et réorganisée. Il se pourrait aussi que cette nouvelle armée devienne un acteur politique important dans la lutte pour le pouvoir dans le centre russe.

74 Mais la Russie n'est plus l'intervenant extérieur exclusif dans la région. Depuis l'implosion de l'Union soviétique, la géostratégie de cette région d'Eurasie a été modifiée. Le Caucase a traditionnellement été une zone de compétition entre trois empires : l'ottoman, le perse et le russe. L'histoire semble se répéter dans un nouveau décor international. Au départ, les nationalistes turcophones, du type d'Elchebey, proliférèrent dans toute la zone, mais à partir du moment où on comprit que la Turquie ne pouvait pas aider effectivement les Azerbaïdjanais contre les Arméniens, et qu'elle n'avait pas le capital pour financer les projets ambitieux de modernisation des élites post-communistes en Asie centrale, l'euphorie pro-turque se trouva quelque peu altérée.

Cependant, la Turquie séculaire sur laquelle les nord-Américains spéculaient également comme pouvoir régional et "pays charnière" reste l'un des outsiders les plus importants dans le nouveau jeu des nations, dans le Caucase comme en Asie centrale. Bien que la Turquie ait elle-même des problèmes intérieurs (la rébellion kurde et le renouveau islamique), elle est appellée à contrebalancer l'influence islamique antioccidentale iranienne dans la région et particulièrement en Azerbaïdjan ${ }^{67}$ et au Tadjikistan $^{68}$. Le Pakistan, l'Arabie Saoudite, Israël, et, de plus en plus, la Chine constituent d'autres intervenants eurasiens plus reculés. La diaspora arménienne d'influence internationale devrait également être mentionnée. L'Occident, et surtout les États-Unis d'Amérique, qui avaient publiquement refusé de reconnaître tout intérêt de sécurité spécial ou exclusif russe en Europe de l'est, ont reconnu de manière pragmatique la "finlandisation" progressive de la région proche de la Russie, fait particulièrement visible dans le Caucase et au Tadjiskistan. Il semble que la nouvelle influence russe dans la zone soit perçue comme une garantie effective contre le cauchemar d'une possible combinaison de l'islam radical et des capacités nucléaires, qui menacerait les intérêts nord-américains au Moyen-Orient.

De manière moins importante, mis à part ces pouvoirs extérieurs, toutes les formules de paix et de trèves au Caucase sont encore basées sur la territorialisation et la séparation selon l'ethnicité, sur une bantoustanisation spécifique de la région, où chaque État-nation, dirigé par sa propre ethnocratie, suivra sa propre ligne de 
développement économique et politique. Paradoxalement, au moment même où le système d'apartheid est démantelé en Afrique du Sud, voilà qu'on l'introduit dans les Balkans et dans le Caucase. L'accord israëlo-palestinien entre Gaza et Jéricho n'est pas vraiment différent, bien qu'il s'agisse là d'une autre histoire dans un autre laboratoire.

\section{Les liens d'une même chaîne}

77 Tout au long de ce texte, j'ai employé le vocabulaire géostratégique, quelque peu dénigré par les sciences sociales dans le passé, mais immédiatement reconnaissable par de nombreux politiciens et journalistes. En refusant le déterminisme organique et environnemental de la geopolitik allemande basée sur la sélection et la suprématie raciales, de nombreuses approches académiques de la guerre froide rejetèrent la géostratégie humaine et politique. Bien qu'on fasse allusion aux influences et aux facteurs externes, la dimension géographique des politiques est également absente des théories de transition contemporaines, incapables d'expliquer pourquoi le pouvoir politique change au sein des États et des groupes au cours du temps, et pourquoi et comment ces changements influencent à leur tour des développements politiques intérieures. Néanmoins, depuis quelques années, nous assistons à un intérêt retrouvé pour la "nouvelle" géopolitique ou géopolinomique (géo-politique-économique), associant la géographie humaine et politique aux théories du développement, à l'économie politique internationale et à l'analyse systémique. Les théories "cycliques", soulignant la périodicité dans l'évolution et les changements de pouvoir, ajoutent une dimension dynamique à cette analyse spatiale statique. Par exemple, pour Modelski et Thomson, les cycles s'apparentent à des "répétitions typiques" qui ont duré de 92 à 129 ans, ces cinq derniers siècles ${ }^{69}$. Gills et Gunder Frank se sont aperçus que les cycles d'accumulation économique correspondaient aux cycles d'hégémonie politique; ainsi, ils ont tracé des phases alternatives de "contraction" et d'“expansion" remontant jusqu'à 1700 avant J.C..$^{70}$ !

Cependant, les deux super-puissances avaient des perceptions plus statiques et plus pragmatiques de leurs intérêts. L'Union soviétique s'appropriait et satellisait de vastes régions en Europe orientale et affirmait fermement sa suprématie militaire au coeur de l'Eurasie ; les États-Unis d'Amérique imposèrent leur hégémonie sur et sous les mers, "maitrisant" l'expansionnisme soviétique. Les deux super-puissances établirent un monde bi-polaire comprenant des domaines stratégiques avec leurs subdivisions régionales, leurs propres hiérarchies, se contrebalançant l'une l'autre à travers l'équilibre obstiné des forces alternantes qui entraîna le système des relations internationales dans près d'un demi siècle de constructions militaires, de transferts d'armes et de conflits locaux et régionaux. "Bien que cette stratégie ait finalement conduit à la ruine de l'ancienne Union soviétique et ait précipité la dissolution de son empire, elle entraîna également les États-Unis dans une guerre stratégiquement fautive et destructrice au Viêt-Nam, et dans une course à l'armement qui devait saper l'économie nord-américaine et affaiblir son tissu social. ${ }^{71}$ La stratégie bi-polaire ellemême, avec tous les paradigmes qui s'y rapportent, la politique d'endiguement et la théorie des dominos, ne signifia plus grand chose dès lors que l'équilibre dynamique de la guerre froide s'effondra.

En ce moment même, dans les Balkans et dans le Caucase, nous souffrons du déséquilibre $\mathrm{du}$ système géopolitique mondial. Les expériences tragiques qui se 
déroulent actuellement dans nos laboratoires contribueront certainement à de nouveaux réalignements régionaux et un réajustement mondial, ouvrant la voie à un nouvel équilibre.

Pour l'instant, il semble que nos ethnocrates, avec leur déterminisme spatial et ethnique à l'état brut et leur interprétation paroissiale de l'histoire, sont plus à même de faire face aux turbulences de la transition du système mondial que de nombreux groupes d'experts universitaires et politiques déphasés par leurs grandes théories dépassées. Comparés aux ethnocrates, les chefs d'États puissants apparaissent parfois comme de pauvres amateurs. Après la fin soudaine de la guerre froide, nos seigneurs de guerre se servirent du nouveau vide au sein du pouvoir pour acquérir le plus de lebensraum possible. Ils sont guidés par des considérations géostratégiques primitives d'un autre âge correspondant à leur propre interprétation provinciale et limitée des relations internationales, alors qu'ils évoluent dans un monde modifié où le contrôle physique de l'espace et la souveraineté territoriale formelle sont moins importants que l'inter-pénétration économique et la libre circulation des capitaux, de la technologie, des produits de base, de la main d'oeuvre et des idées. Néanmoins, ils n'ont pas complètement tort, car ils sentent ce qui manque, les discontinuités, les rivalités et les contradictions existant au sein du système mondial qu'ils essayent d'exploiter. Ils espèrent également que leurs futurs statuts et rangs internationaux dépendront effectivement de leur localisation, leur taille, des ressources naturelles, des potentiels économiques et humains, de la force militaire des territoires qu'ils contrôlent, et, là encore, ils n'ont pas tort, car la hiérarchie internationale des États-nations est précisément déterminée par de tels critères. Le paradoxe est que les élites politiques des États-nations très industrialisés et économiquement puissants, censés être guidés uniquement par l'économie politique du système mondial qu'ils sont en train de construire, n'ont rien trouvé d'autre que d'utiliser des techniques géostratégiques aussi dépassées pour promouvoir et protéger leurs intérêts dans nos deux laboratoires (la fragmentation territoriale afin de réduire le poids des unités territoriales plus importantes, les politiques consistant à diviser pour mieux régner, la création de zones tampons, d'États-tampons et de régions passage, la redéfinition de zones d'influence, l'utilisation de certains États comme "charnière" et d'autres comme boucs émissaires, les sanctions économiques, la coercition politique par le biais d'organisations interétatiques, la menace de la violence, l'usage de la force, etc.). La contradiction entre les processus de mondialisation et d'intégration d'une part, et de la fragmentation de l'Europe de l'est et de l'ex-Union soviétique d'autre part, n'est qu'apparente : en fait, elles représentent deux aspects du même processus mondial ${ }^{72}$.

81 Finalement, les ethnocrates de nos régions auront à faire leur choix entre l'intégration de leurs États-nations dans le système mondial et leur isolation. S'ils optent pour leur intégration et s'ils sont acceptés, ils seront effectivement récompensés, peut-être même honorés comme des chefs d'État ou des personnalités, mais leurs pays dévastés tant sur le plan humain que matériel seront réduits au rang d'États-clients ou de républiques bananières. S'ils refusent de jouer le jeu, ils seront bannis comme la junte militaire birmane ou comme l'Irak de Saddam Hussein. Pour survivre, la plupart d'entre eux devront choisir l'intégration et, malheureusement, le seul article commercial à leur disposition sera justement la nationalité dont ils avaient l'intention de promouvoir les intérêts "souverains", "vitaux" et "historiques" et qu'ils ont personnellement utilisé dans des guerres autodestructrices. C'est exactement le cas de la Serbie de Milošević imposant à présent des "sanctions" à l'encontre des Bosniaques 
serbes qui ont l'impression d'être "trahis". Bien sûr, ils ont été manipulés sans aucun scrupule par leurs propres seigneurs de guerre, puis par Milošević, qui a récemment effectué un revirement total pour rester au pouvoir. De belliqueux, il est brusquement devenu pacificateur et, parce qu'il n'a aucune opposition crédible pour promouvoir la paix encouragée par la communauté internationale, il est le seul facteur, côté serbe, susceptible de garantir le bon déroulement des événements. Milošević pourrait bien survivre et même décrocher le prix Nobel de la paix, mais le corps national serbe restera mutilé pour de nombreuses années. Il en va de même pour Tudjman, le "père de la nation croate", dont "l'accomplissement historique" se traduisit par l'occupation de plus de $20 \%$ du territoire de l'ancienne République socialiste de Croatie avec 690000 réfugiés et personnes déplacées, et la perte de peut-être près d'un demi million d'habitants (Serbes et émigrés). Rien qu'en Bosnie-Herzégovine, on dénombre officiellement 2280000 réfugiés, ce qui signifie que pratiquement un habitant sur deux de l'actuel pays divisé a été victime de la guerre. Le nombre de tués, pour la plupart des civils, doit probablement atteindre 300 000. La Serbie et le Monténégro ont enregistré 545000 réfugiés. Quant aux ex-Yougoslaves qui ont pu échapper à la guerre, ils seraient environ un demi million à parcourir le monde. Je suis l'un d'entre eux. Ces vagues d'émigration sont différentes des précédentes dans le sens où celles-ci comprennent un grand pourcentage de jeunes gens lettrés qui fuyaient le service militaire. Le reste se compose d'un personnel hautement qualifié, chassé par l'insécurité et la pauvreté grandissante. Il s'agit là d'un tragique exode de cerveaux qui a déjà dévasté les élites "nationales" existantes, qui continue à l'heure actuelle et qui n'annonce rien de bon pour le futur. L'ironie de l'histoire, c'est que Milošević, Tudjman, Izetbegović et leurs acolytes qui, par leurs programmes "nationaux" respectifs exclusifs, ont conduit à la guerre et à l'actuel bain de sang, sont maintenant appelés à devenir des agents de la paix. L'histoire nous a déjà fournit des exemples identiques dans le passé et je pourrais citer ici Quisling, Pavelić ou Monseigneur Tiso, qui ont sacrifié volontairement leur propre nation pour des idéologies "nationales" douteuses et paroissiales. Cette version du nationalisme a toujours été à l'encontre du but recherché.

Il se passe exactement la même chose dans le laboratoire du Caucase qui semble fonctionner comme un vase communicant avec les Balkans. Selon le rapport du Haut Commissariat aux Réfugiés des Nations Unies, le nombre officiel de personnes déplacées en Azerbaïdjan s'élève à 550 000, 330000 en Arménie et 146000 en Géorgie, auquel on peut ajouter un million de réfugiés éparpillés et des retours "volontaires" dans d'autres parties de la CEI. Ter-Petrossian et Aliev, les Présidents arménien et azerbaïdjanais, pourraient bien se mettre d'accord sur la formule qui mettra fin à la situation actuelle de ni-guerre ni-paix, mais cela ne pourra pas remédier aux catastrophes humaines causées par les politiques de nettoyage ethnique. C'est également valable pour la relation entre Tbilissi, l'Abkhazie et l'Ossétie du sud séparatistes, et il ne faut pas oublier les perpétuels conflits de clans géorgiens entre eux. Le tissu social de toutes ces communautés a été endommagé et leurs économies brisées. Même si la paix et la stabilité sont restaurées, il faudra des années avant qu'on ne puisse espérer une vie meilleure.

Le paradoxe entre les deux histoires, c'est que beaucoup de gens, y compris ceux qui ont volontairement pris part à la guerre, opteraient, si on leur laissait le choix, pour l'émigration au Canada, en Australie ou dans n'importe quel autre endroit convenable susceptible de leur offrir la sécurité, du travail et un autre niveau de vie. Il doit y avoir peu de monde préférant rester dans sa patrie "nationale" estropiée. Cependant, si on 
leur posait la question, tous ceux qui ont été obligés de quitter leur maison et d'abandonner leurs biens pour aller dans des pays "étrangers" reviendraient chez eux, s'il y avait certaines garanties fondamentales contre la discrimination. Si la normalité était restaurée dans un contexte démocratique, c'est-à-dire si la loi était appliquée et les frontières ouvertes à la libre circulation des marchandises, des personnes et des idées, les individus iraient inévitablement là où ils pourraient trouver du travail, des logements, des écoles et des soins médicaux, et nos populations seraient encore une fois mélangées. Si cela pouvait arriver, le melting-pot ethnique d'avant-guerre serait restauré en un rien de temps, et la question à se poser serait : quelle est la rationalité de toutes ces guerres, ces souffrances et ces destructions? La réponse s'impose d'ellemême : les seules personnes qui en ont bénéficié sont nos ethnocrates. Pour eux, la guerre était un moyen d'ascension sociale. En quelques années, ils ont acquis richesse et statut social. Du jour au lendemain, ils sont devenus ministres, parlementaires, généraux, juges, ambassadeurs et, le plus important de tout, les propriétaires de nos pays. Le reste d'entre nous, nos propres nations, en ont payé le prix. Avec bon espoir, d'ici une ou deux décennies, nos sociétés se civiliseront, et nos pays réussiront à bâtir des économies de marché et de véritables démocraties. Alors, nous serons mûrs pour l'intégration dans le système mondial et nous prendrons, en marche, le train européen en tant que régions d'un tout étendu. La question légitime que nous poseront nos descendants sera : pourquoi a-t-on mis tant de temps à rejoindre l'Europe et pourquoi nos pères et mères se sont lancés dans des guerres aussi stupides? Si jamais je suis toujours là, je leur répondrai que, malheureusement, il y avait une profonde logique dans tout cela.

Mais nos laboratoires régionaux ne sont pas isolés... Il y a d'autres expériences, certes moins dramatiques, qui se déroulent dans d'autres régions eurasiennes. Chacune d'entre elles se compose d'un regroupement d'États-nations post-soviétiques dont le statut international n'a pas encore été bien déterminé et qui doivent également faire front aux problèmes intérieures de la transition à l'économie de marché et à la démocratie. Le groupe Visegrád constitue un autre laboratoire et il semble que la Pologne, la République tchèque, la Slovaquie et la Hongrie fassent partie d'un aménagement global différent de celui des Balkans. Les trois pays baltes, l'Estonie, la Lituanie et la Lettonie semblent suivre un autre scénario, s'alignant sur le modèle scandinave. L'Ukraine et la Biélorussie sont des cas spéciaux s'inscrivant dans les "régions proches" de la Russie. Leurs relations avec la Russie sera en grande partie déterminante dans l'avenir de l'ancien espace soviétique. On a déjà abordé le sujet de l'Asie centrale et nous pourrions aussi procéder de la même façon de laboratoire en laboratoire. Dans leur ensemble, ils constituent les liens d'une même chaîne.

\section{NOTES}

1. Toutefois, quelques références doivent être mentionnées : Tilly (Charles), ed., The Formation of Nation States in Europe, Princeton : Princeton University Press, 1975 ; Eisenstein (S. N.), Rokkan (Stein), Building States and Nations, Beverly Hills : Sage, 1973 ; Geertz (C.), Old Societies and New 
States, New York: Collier-Methuen, 1963 ; Poulantzas (Nikola), Political Power and Social Classes, London : New Left Books, 1973 ; Giddens (Anthony), The Nation-State and Violence, Berkeley / Los Angeles : University of California Press, 1985.

2. Carl (F.), Brzezinski (Zbigniew), Totalitarian Dictatorship and Autocracy, Cambridge : Harvard University Press, 1956, pp. 9-10.

3. Cité in Ash (T. G.), The Use of Adversity, London : Hutchinson, 1966.

4. Pusić (Vesna), "Diktatura s demokratskim legitimetetom» (La dictature à la légitimité démocratique), Erasmus, (1), april 1993.

5. Le modèle soviétique en développement a toujours été une référence négative ou positive pour les autres Partis communistes. Le terme de Brejnev de "véritable socialisme" entra donc dans le vocabulaire politique courant et fut utilisé en Europe de l'Est pour décrire tous les régimes communistes européens.

6. Une telle approche m'a été inspirée par J. Linz, qui établit une distinction entre l'Espagne de Franco, l'Allemagne d'Hitler et l'Italie de Mussolini. Cf. Linz (Juan J.), «An Authoritarian Regime: Spain», inAllardt (Eric), Littunen (Y.), eds., Cleavages, Ideologies and Party Systems, Helsinki : Westmark Society, 1964.

7. En écrivant à propos des tendances politiques contemporaines en Albanie, F. Tarifa affirme que « le système politique nouvellement établi dans ce pays, bien que formellement pluraliste, ressemble plus au vieux modèle communiste du parti unique. Le parti au gouvernement, bien qu'anti-communiste, est, comme aux jours anciens, "aux commandes". Du sectarisme stalinien, il est passé à un sectarisme "démocratique". Il a remplacé le communisme en tant qu'idéologie par une autre idéologie qui pourrait être décrite comme du "démocratisme". Strictement identique au marxisme, c'est devenu une idéologie étatique hégémonique, représentant une tentative officielle pour subordonner les valeurs culturelles aux buts politiques, et pour servir d'instrument dans la recherche du contrôle du pouvoir " (Tarifa (F.), "Albania's PostCommunist Transition », Balkan Forum, 1 (5), december 1993, pp. 130-131). Voir aussi Zogaj (P.), «Learning from the Past », East European Reporter, 5 (5), september-october 1992, p. 65.

8. La Tchécoslovaquie (en fait la région de Bohème) était atypique, car il s'agissait du seul pays industriellement développé d'Europe de l'Est. Le processus de collectivisation a été renversé en Pologne et en Yougoslavie dans les années 1950. Cependant, la Yougoslavie a réussi à construire un secteur agro-industriel efficace sur environ $12 \%$ de la surface cultivable et dont la production de produits de base a été capable de satisfaire la demande locale.

9. L'"égalisation" ou l'“harmonisation" proclamée du niveau de développement économique n'a jamais été réalisée. Pour certains territoires, comme la province du Kosovo en Serbie ou la région du Nagorno Karabagh en Azerbaïdjan, le fossé s'est même élargi.

10. in Chirots (D.), ed., The Crisis of Leninism and the Decline of the Left, Seattle: University of Washington Press, 1991, p. 6. Pour une vision générale de la relation entre technologie, économie et société, voir : Poznanski (Kazimierz), Technology, Competition and the Soviet Bloc in World Trade, Princeton: Princeton University Press, 1980 ; Stewart (F.), Technology and Underdevelopment, London: Macmillan, 1978; Sucliffe (R. B.), Industry and Underdevelopment, London: Addison Wesley, 1972 ; Landes (S.), The Unbound Prometeus : Technological Change and Industrial Development in Western Europe from 1750 to the Present, Cambridge : Cambridge University Press, 1969 ; Rostow (W. W.), Stages of Economic Growth : A Non-Communist Manifesto, Cambridge : Cambridge University Press, 1960.

11. Batt (Judy), «The Politics of Economic Transition », in White (S.), Batt (J.), Lewis (P.), ed., Developments in East European Politics, Durham : Duke University Press, 1993.

12. Kaminski (Bartlomiej), The Collapse of State Socialism. The Case of Poland, Princeton : Princeton University Press, 1991, pp. 24, 42, 43.

13. Young (B.), « With Axes in their Eyes : Rentierism and Market Reform in Yugoslavia », Studies in Comparative Communism, 25 (3), september 1990. 
14. S. Tarrow dans son livre parle de «bureaucratie territoriale» (cf. Tarrow (S.), Between Center and Periphery, Yale: Yale University Press, 1977, p. 77). Pour une bonne esquisse des relations centre-périphérie au sein de la fédération soviétique, voir Gleason (Gregory), Federalism and Nationalism: The Struggle for Republican Rights in the USSR, Boulder: Westview Press, 1990, pp. 81-101.

15. Le processus de décomposition de l'URSS a été particulièrement bien décrit par Carrère d'Encausse (Hélène), La gloire des nations ou la fin de l'empire soviétique, Paris : fayard, 1990. Voir aussi Holmes (L.), The End of Communist Power: Anticorruption Campaign and Legitimation Crisis, Cambridge : Polity Press, 1993, et Walker (R.), Six Years Which Shook the World: Perestroika - The Impossible Project, New York / Manchester : Manchester University Press, 1993.

16. L'équipe de recherche de l'École d'Économie de Varsovie est venue à la même conclusion : Markowska (E.), Szostak (M.), State and Development. Towards a Reappraisal, Warsaw : Warsaw School of Economics, Research Institute for Developing Economies, 1993 ; Brzezinski (Zbigniew), Out of Control. Global Turmoil on the Eve of the $21^{\text {st }}$ Century, New York: Collier Books, Macmillan Publishumg Co., 1993, bien que leurs approches respectives soient différentes.

17. Giddens (Anthony), op.cit., p. 120.

18. Ibid., p. 289.

19. Wiarda (H. J.), Introduction to Comparative Politics. Concepts and Processes, Balmont: Wadsworth Publishing Co., 1993, p. 77.

20. Cf. Cohen (J.) et al., « De-Parsonizing Weber : A Critique of Parsons' Interpretation of Weber Sociology », American Sociological Review, 40, april 1975.

21. Weber (Max), General Economic History, New Brunswick : Transaction Books, 1981, p. 339.

22. Nairn (Tom), The Break-Up of Britain, London : New Left Books, 1977, p. 353.

23. Skocpol (T.), Bringing the State Back In, Cambridge : Cambridge University Press, 1985.

24. Cf. Callaghy (T. M.), "The State and Development of Capitalism in Africa: Theoretical, Historical and Comparative Reflection », in Rotchild (D.), Chazan (N.), eds., The Precarious Balance. State and Society in Africa, Boulder / London : Westview Press, 1988.

25. Szporluk (Roman), Communism and Nationalism: Karl Marx versus Friedrich List, New York: Oxford University Press, 1988; Senghas (D.), «Friedrich List and the Basic Problems of Modern Development ", Review, 14 (3), Summer 1991.

26. Weber (Max), «Politics as a Vocation », in Gerth (H. H.), Mills (C. W.), ed., From Max Weber : Essays in Sociology, London : Routledge \& Kegan Paul, 1970, p. 77.

27. Horvat (Branko), « Društveno vlasništvo u Hrvatskoj» (La propriété sociale en Croatie), texte non publié, sans date.

28. Smith (Anthony D.), National Identity, London : Penguin Books, 1991, pp. 9-13.

29. Williams (Colin H.), Smith (Anthony D.), "The National Construction of Social Space", Progress in Human Geography, 7, 1983 ; Murphy (Alexander B.), «Regions as Social Constructs », Progress in Human Geography, 15, 1991. Le travail de Rossburg et Jackson fournit une étude séminale sur le rôle du régime d'étatisation juridique dans la protection des États faibles qui à leur tour se servent des politiques d'identité pour établir une base de pouvoir et une fidélité au sein de la société.

30. Mostov (July), « Constituting Ethnocracy », texte non publié, sans date.

31. Mostov (July), «Democracy and Politics of National Identity », Studies in European Thought, 45, january 1994.

32. Gellner (Ernest), Nations et nationalisme, Paris : Payot, 1989, pp. 90-95.

33. Ibid., p. 76.

34. Ibid., p. 72.

35. Voir l'excellent recueil d'études réunies dans Gross (J. A.), ed., Muslims in Central Asia. Expressions of Identity and Change, Durham : Duke University Press, 1992. À propos des différents niveaux de l'auto-identification nationale dans les États successeurs de l'URSS, R. Taras parle de 
"nationalisme gigogne" (in Bremmer (Ian), Taras (Ray), eds., Nations and Politics in the Soviet Successor States, Cambridge : Cambridge University Press, 1993, pp. 513-538).

36. H. Carrère d'Encausse (Carrère d'Encausse (Hélène), op.cit.) semble croire que les nations sont des catégories « données par Dieu », supprimées en bloc par le communisme.

37. Cf. Nekrich (A. M.), The Punished Peoples: The Deportation and Tragic Fate of Soviet Minorities at the End of the Second World War, New York: W. W. Norton, 1978. Cependant, dans la politique de terreur hasardeuse de Staline, les nationalités étaient visées au même titre que d'autres grandes catégories, comme les "trotskistes", les “koulaks", les "prêtres", les “officiers militaires", les "prisonniers de guerre", les "médecins", etc.

38. Il y a beaucoup de définitions de la nation. Une des plus concises, qui a eu des conséquences de portée considérable, a été formulée par Staline qui a défini la nation comme «une communauté stable, historiquement constituée, de langue, de territoire, de vie économique et de formation psychique, qui se traduit dans la communauté de culture» (Staline (Joseph), «Le marxisme et la question nationale ", Le marxisme et la question nationale et coloniale, Paris : Éditions sociales, 1949, p. 15). Muriel Atkin définit la nationalité comme « une forme politique inhérente à l'organisation sociale, dont les partisans donnent la primauté aux intérêts de la nationalité (intérêts définis par ces mêmes partisans) et déterminent les buts politiques et culturels du groupe, et, dans certains cas, les dimensions de l'État » (in Gross (J. A.), ed., op. cit., p. 47).

39. Hroch (Miroslav), «From National Movement to the Fully-Formed Nations, the NationBuilding Process in Europe », New Left Review, (198), march-april 1993.

40. Royce (A. P.), Ethnic Identity, Bloomington : Indiana University Press, 1982, p. 185.

41. Gellner (Ernest), op.cit., p. 137-138.

42. Ibid., p. 159-160.

43. in Bremmer (Ian), Taras (Ray), eds., op.cit.

44. Tamir (Yeal), Liberal Nationalism, Princeton: Princeton University Press, 1993, pp. 64-65, 83-85.

45. Cité in Haupt (Georges), Lowy (Michael), Weill (Claudie), éds., Les marxistes et la question nationale, François Paris : Maspero, 1974, p. 116.

46. Lénine (Vladimir I.), Notes critiques sur la question nationale / Du droit des peuples à disposer d'eux-mêmes, Paris : Éditions sociales, 1952, p. 39.

47. Harman (c.), «The Return of the National Question », International Socialism, (56), autumn 1992.

48. Cohen (B), "Geopolitics in the New World Era : A New Perspective for an Old Discipline », in Demko (G. J.), Wood (W. B.), eds., Reordering the World, Boulder : Westview Press, 1994, p. 20.

49. Schumpeter (J. A.), Capitalism, Socialism and Democracy, New York: Harper and Row, 1950 (3ème éd.), et The Theory of Economic Development : An Inquiry into Profits, Capital, Credits, Interests, and the Business Cycles, New York : Oxford University Press, 1961.

50. Gilpin (Robert), The Political Economy of International Relations, Princeton: Princeton University Press, 1987.

51. Ibid., pp. 343-344.

52. Ibid., p. 346.

53. Ibid., p. 345.

54. Aggarval (Vinold), Liberal Protectionism: The International Politics of Organized Textile Trade, Berkeley : University of California Press, 1985.

55. Le célèbre article de F. Fukuyama, "The End of History » (National Interest, Summer 1989), utilisant des approches idéologiques et des lexiques politiques opposés, et le programme du PCUS (Parti Communiste de l'Union Soviétique) de 1961, prédisant le dépassement économique par de l'Union soviétique des États-Unis d'Amérique dans les 20 ans, sont pratiquement symétriques. Cependant, dans un livre ultérieur plus élaboré, The End of History and the Last Man (New York: Avon Books, 1992), Fukuyama est revenu sur certaines de ses conclusions. 
56. Hobsbawm (Eric), « The Crisis of Today's Ideologies », New Left Review, (192), 1992.

57. Popularisé très récemment par l'article de Kaplan (Robert), "The Coming Anarchy" (Atlantic Monthly, February 1994), la littérature post-moderniste du chaos prend de plus en plus d'importance. Cf. Glieck (J.), Chaos, New York: Penguin Books, 1987 ; Baumol (W.), et. al., "Chaos: Significance, Mechanism and Economic Application», Journal of Economic Perspectives, (3), 1989 ; Berge (P.), et. al., Order Within Chaos, New York : John Wiley, 1984.

58. Pour le concept, qui pourrait aussi bien être appliqué à l'État grec contemporain, cf. Cohen (S. B.), « Assymetrical States and Global Political Equilibrium », SAIS Review, 4 (2), 1984.

59. D'un autre côté, il est également vrai que la solidarité avec les "frères orthodoxes serbes" est un problème majeur dans la politique intérieure russe. C'est devenu le cri de ralliement de l'opposition rouge-brune. La cooptation de la Russie dans le groupe de contact devait soulager cette pression sur le gouvernement d'Eltsine.

60. Si demain la marine russe entre dans les Bouches de Kotor pour "assurer la paix", le testament de Pierre le Grand sera réalisé! Un dénouement paradoxal que les pouvoirs occidentaux ont essayé d'empêcher pendant des siècles.

61. Brzezinski (Zbigniew), «Premature Partnership », Balkan Forum, 2 (2), june 1994.

62. Brzezinski (Zbigniew), Out of Control (op.cit.).

63. Dans «The View From The Outside ", M. Sahair décrit le sort du $366^{\text {ème }}$ régiment de l'armée soviétique (Sahair (M.), Khidjaly : Its Last Days, Baku : Publishing House Azerbaijan, 1992).

64. Cf. Omrod (J.), «The Northern Caucasus : Fragmentation of Federation? ", in Bremmer (Ian), Taras (Ray), eds., op.cit. et Twining (D. T.), The New Eurasia. A Guide to the Republics of the Former Soviet Union, Wesport, Conn. : Praeger, 1993, pp. 121-123, 129, 134-135.

65. L'un d'entre eux, Rouslan Khasboulatov, tchétchène et président du Parlement russe, et, à l'époque, un des ennemis d'Eltsine.

66. Incarné dans le tandem de Pavel Gratchev, ministre de la Défense et Viktor Tchernomyrdine, Premier ministre.

67. La plupart des Azerbaïdjanais sont chi'ites et environ 9 millions d'Azéris vivent en Iran, où ils représentent la seconde ethnie après la majorité perse. On peut trouver des renseignements utiles sur la religion et les minorités ethniques dans le "voisinage" du Caucase et de l'Asie centrale dans Banuazizi (A.), Weiner (Myron), eds., The State, Religion, and Politics. Afghanistan, Iran, and Pakistan, Syracuse : Syracuse University Press, 1986.

68. Les Tadjiks sont de souche iranienne, bien qu'ils soient sunnites. Les Tadjiks (ou Daris ou Parziwans) sont aussi un groupe ethnique important en Afghanistan où ils représentent environ $1 / 5^{\text {ème }}$ de la population totale. Leur nombre est estimé à 3,5 millions.

69. Modelski (G.), Thomson (W. R.), Sea-Power in Global Politics, 1494-1983, London : Macmillan, 1988 ; Modelski (G.), Long Cycles in Modern World Politics, Seattle : University of Washington Press, 1987.

70. Gills (B. K.), Frank (A. Gunder), « World System Cycles, Crisis, and Hegemonical Shifts, 1700 $\mathrm{BC}$ to $1700 \mathrm{AD} »$, Review, 15 (4), fall 1992.

71. Cohen (S. B.), art.cit.

72. Iveković (Rada), texte non publié, sans date. 


\section{RÉSUMÉS}

Dans cet article, la Yougoslavie et le Caucase servent à illustrer l'analyse des relations entre l'ethnicité, le nationalisme, l'État-nation, l'économie politique globale et la hiérarchie changeante de la puissance internationale. Ces deux espaces forment des «laboratoires régionaux » affrontant des problèmes similaires. Les seuls à avoir bénéficier des guerres qui s'y déroulent sont les ethnocrates.

In this article, Yugoslavia and Transcaucasia are instances which illustrate the analysis of relationship between ethnicity, nationalism, nation-state, the global political economy and the changing hierarchy of international power. These two spaces are « regional laboratories » which are facing similar problems. Ethnocrats are the only ones who have benefited from the wars in these regions.

\section{AUTEURS}

\section{IVAN IVEKOVIĆ}

I. Iveković est professeur de science politique à l'Université Américaine du Caire. 